\title{
Effective Biomarkers and Therapeutic Targets of Nerve-Immunity Interaction in The Treatment of Depression: A Integrated Analysis of miRNA-mRNA Regulatory Networks
}

\section{Zi-xuan Wu}

Guangzhou University of Traditional Chinese Medicine: Guangzhou University of Chinese Medicine

\section{Xuyan Huang}

Guangzhou University of Traditional Chinese Medicine: Guangzhou University of Chinese Medicine Min-jie Cai

Guangzhou University of Traditional Chinese Medicine: Guangzhou University of Chinese Medicine Peidong Huang ( $\square$ yeruyun@163.com )

Yunnan University of Traditional Chinese Medicine https://orcid.org/0000-0002-3044-9269

\section{Zunhui Guan}

Kunming Municipal Hospital of Traditional Chinese Medicine

\section{Research}

Keywords: Major depressive disorder (MMD), MicroRNAs (MiRNAs), DE-miRNAs, Regulatory Networks, Bioinformatics

Posted Date: October 12th, 2021

DOl: https://doi.org/10.21203/rs.3.rs-958945/v1

License: (c) (1) This work is licensed under a Creative Commons Attribution 4.0 International License. Read Full License 


\section{Abstract}

Background: Major depressive disorder (MDD) is an emotional disorder that has a negative effect on patients' studies and daily lives. A great number of studies have found that miRNAs play an important role in the development of MDD and that they can be used as a biomarker for the diagnosis and treatment of MDD. However, there have been few investigations on nerve-immunity interaction therapy for MMD patients' brains.

Methods: We attempted to evaluate MDD in the gene expression matrix database and miRNAs in plasma samples from healthy controls using bioinformatics methods. Four plasma miRNAs (DE-miRNAs) samples were found from MDD patients. Funrich planned the transcription factors and target genes of miRNAs, and the enrichment of TF and GO was examined. The intersecting mRNAs were discovered by comparing the various expressions of the projected target genes and 5 mRNAs (DE-mRNAs) samples. In the end, 34 DE-miRNAs, 386 DE-mRNAs, and 17 intersecting mRNAs were detected. Intersecting core genes were then investigated using GO and KEGG enrichment analysis to find the intersecting mRNA. Identify particular candidate genes and pathways in neurology and immunology that may be associated with MDD for further investigation.

Results: We discovered 17 important HUB genes by the advance of a miRNA-mRNA network, and 5 HUB DE-MRNAs were derived following CytoNCA topology.

Conclusion: Our findings from a comprehensive bioinformatics analysis of miRNAs and mRNAs in MDD show that DE-miRNAs like miR-338-3P and miR-206 may be excellent biomarkers and potential therapeutic targets for the treatment of MDD via nerve-immunity interaction.

\section{Introduction}

Major depressive disorder (MDD) is a crippling mental illness defined by depression and emotional disturbance induced by a variety of psychological variables $\left[{ }^{1}\right]$. The high prevalence rate, recurrence rate, and disability rate is all tough issues to address the prevention and treatment of $\operatorname{MDD}\left[{ }^{2]}\right.$. At the moment, at least $20 \%$ of the world's populations is affected by MMD, and the WHO projects that the disease burden of MDD will surpass that of heart disease by $2030[3-[\mathrm{a} a \mathrm{a} a]$. The occurrence of MDD not only decreases patients' quality of life, but also places a significant cost on the family and society ${ }^{5]}$. Monoamine transmitter, immune-inflammation, stress, neuroscientist, and other popular ideas of MDD are now under investigation ${ }^{6]}$. Scholars have paid increasing emphasis in recent years to research on the pathophysiology of immunity-inflammation and $\operatorname{MDD}[$ [-] $[$ a8a]. Depending on several research, the activation of the peripheral immune system is linked to $\operatorname{MDD}\left[{ }^{[9]}\right.$. However, the pathobiology behind MDD inflammation is not well known, and more study is needed to uncover precise diagnostic biomarkers. The interplay of chronic moderate stress, neuroinflammation, and immune response alterations has been demonstrated to have a role in the pathogenesis of $\operatorname{MDD}\left[{ }^{10]}\right.$. More and more research has revealed that the neurological system is inseparable from the immune system. 
Currently, chemical medications are utilized mostly in the clinical treatment of MMD in Western medicine, whereas Chinese material media, acupuncture, and moxibustion are frequently used in traditional Chinese medicine (TCM) ${ }^{[1]}$. Traditional antidepressants, on the other hand, can only relieve part of the symptoms of MMD patients, and $20 \%$ to $30 \%$ of MMD patients do not react to submit antidepressant medications. Neuroinflammation is seen as one of the causes of treatment ineffectiveness[ ${ }^{12]}$. After an injury, neuroinflammation is the result of an imbalance in the production and release of pro-inflammatory and anti-inflammatory cytokines from central or peripheral sources $\left[{ }^{13}\right]$. The most noticeable aspect of neuroinflammation is microglia activation. Microglia activation results in the production of nutritional and anti-inflammatory substances under physiological settings. In pathological conditions, such as chronic stress or infection, microglia become over-estimated, resulting in not only higher amounts of inflammatory chemicals in the brain, but also neuronal damage and death $\left[{ }^{14]}\right.$. Therefore, identifying particular diagnostic biomarkers from the perspective of nerve-immunity interaction in order to effectively manage neuroinflammation and restore the function of neurotropism and neurotransmitters is critical to basic or clinical MMD research.

MicroRNAs (MiRNAs) are a type of endogenous intracellular tiny yet non-coding RNA. It can regulate biological processes such as cell proliferation, division, apoptosis, and metabolism as a regulator of many biological processes by encouraging the degradation of mRNAs and blocking their translation, so that its targeted mRNAs play a negative feedback regulation role[ ${ }^{15]}$. MiRNAs have been examined as a potential therapeutic target for a variety of central nervous system illnesses since they are involved in practically all fundamental cellular activities. It is well established that changes in miRNA expression are linked to the pathophysiology of several neurodegenerative illnesses and have significant therapeutic potential in the treatment of mood disorders, including clinical MDD[16-[aa17a[i]][a18a]. Detecting changes in the level of pathogenic miRNAs in the brain will be a very good technique to identify patients with early MMD. Bioinformatics is an important instrument for advancing biological understanding and therapeutic development ${ }^{[9]}$. Therefore, the purpose of this study is to analyze and synthesize the evidence of immunity or of nerve-related miRNA in MDD plasma using bioinformatics, which is important for future fundamental research and clinical application of nerve-immunity interaction therapy of MDD. Figure 1 depicts a summary of this strategy.

[[i]] Lauren Allen,Yogesh Dwivedi. MicroRNA mediators of early life stress vulnerability to depression and suicidal behavior[J]. Molecular Psychiatry,2020,25(2):

\section{Materials And Methods}

\subsection{Data processing of DE-miRNAs}

We select data-sets from the Gene Expression Omnibus (GEO, https://www.ncbi.nlm.nih.gov/geo/). Strategy for searching ('depression' [MeSH] and miRNA [All Fields] and normal) AND ('Homo sapiens' [Organism] AND 'Non-coding RNA profiling by array' [Filter]). The following was the inclusion criteria: Plasma miRNA levels in MMD patients and healthy persons. Finally, four sets of MMD miRNA expression 
profile data were compiled. The data-set GSE5 8105 is based on the GPL18743 platform; the data-set GSE81152 is based on the GPL21814 platform; the data-set GSE152267 is based on the GPL21572124634 platform; and the data-set GSE182194 is based on the GPL24741 platform, and it contains 88 MMD samples and 42 normal samples. The four miRNA chip data sets mentioned above are merged by perl software, and the VENN package of the four miRNA chip data sets are formed (Figure 2) in the R4.1.0 VENN package (https://cran.r-project.org/web/packages/venn/). The R4.1.0 Sva and Limma are used to multi-chip data-set for data rectification (batchNormalize). Genes acquired by the Limma software were determined to be significantly differentially expressed miRNAs using the corrected miRNA chip data-set and log2 (fold change) $>2$ or log2 (fold change) $<-2$ as screening thresholds (DE-miRNAs). In R4.1.0, the pheatmap package (https://cran.r-project.org/web/packages/pheatmap/) is used to construct DEGS heat maps and volcanoes.

\subsection{Identification of TF and targeted genes of DE-miRNAs}

The FunRich3.1.3 software is used to annotate DEMs' GO function, which includes annotations of pathways and transporters (TF), as well as biological processes (BP), cellular components (CC), molecular functions (MF), etc[20]. FunRich was used to classify miRNAs with statistical significance, upregulation, and down-regulation in this work, and the first 10 transporters and target genes of DE-miRNAs were found.

\subsection{Data processing of DE-mRNAs}

Choosing a GEO data-set from the database. ('depression' [MeSH] and mRNA [All Fields] and normal) AND ('Homo sapiens' [Organism] AND 'Expression profiling by array' [Filter]). The following were the inclusion criteria: MMD sufferers' or healthy people's plasma mRNA Finally, five sets of MMD mRNA expression profile data were acquired. The data-set GSE19738 is based on the GPL6848-9572 platform, while the data-sets GSE32280, GSE44593, GSE53987, and GSE98793 are based on the GPL570-55999 platform, and include 80 MMD samples and 175 normal samples. Perl software is used to integrate the aforementioned five mRNA chip data-sets, and VENN diagrams of the five mRNA chip data-sets are created using the R4.1.0 VENN package (Figure 3). The Sva and Limma of R4.1.0 were then employed exclusively for multi-chip data-set data rectification (batchNormalize). Genes acquired by the Limma package were determined to be significantly differentially expressed mRNA using the corrected mRNA data-set, with $\mathrm{P}<0.05$ and log2(fold change) $>2$ or log2(fold change) $<2$ as screening conditions (DEmRNAs). The pheatmap package in $\mathrm{R}$ software is used to construct DEGS heat maps and volcanoes.

\subsection{Intersection genes of target mRNAs and DE-mRNAs}

Given the negative regulation relationship between miRNA and target genes, perl software intersects the target genes up-regulated DE-miRNAs and down-regulated DE-mRNAs, and the target genes downregulated DE-miRNAs are intersected with up-regulated DE-mRNAs, and the intersection genes are defined as core genes. The target genes of DE-miRNAs predicted by FunRich were then compared to DE-miRNAs 
forecast by GEO. The crossing genes were represented by a Venn diagram, and overlapping mRNAs were discovered.

1.5 Construction of miRNA-mRNA network and Protein-protein interaction network

Cytoscape3.7.2 is utilized to build the miRNA-mRNA network of mRNAs[21]. TThe String online tool (https://string-db.org/)[22] is used to create the PPI network of aberrant mRNA. With the protein type "Homo sapiens" and the highest level of confidence (0.150). The PPI network model is then created by Cytoscapee3.7.2.

1.6 Go and KEGG Enrichment analysis of mirNA-mrna regulatory network.

Following the acquisition of the core target, the ClusterProfiler, Colorspace, stringi, ggplot2, DOSE, enrichplot, and org. The R4.1.0 Hs.eg.db was utilized to examine the enrichment of GO and KEGG of the core target. These seven packages can be obtained from bioconductor (http://www.bioconductor.org/). GO (http://geneontology.org/) enrichment primarily examines the target's biological process, cell composition, and the molecular function, whereas KEEG (https://www.kegg.jp/) enrichment examines the target's potential biological pathways and activities.

\subsection{Identification of potential Hub mRNAs and hub miRNAs}

Based on the PPI network obtained above, according to the topological characteristics of the network, three most important parameters were selected by CytoNCA plug-in to screen the Hub mRNAs: Degree Centrality (DC) $\left[{ }^{23]}\right.$, Closeness Centrality (CC) $\left[{ }^{24]}\right.$, and Betweenness Centrality (BC) $\left[{ }^{25]}\right.$. DC relates to the number of other nodes associated with a node in the network. The higher the degree centrality, the greater the importance of the node. BC estimates the number of shortest paths through a node. The more the number of shortest paths through a node, the higher its intermediary centrality. CC calculates the sum of the distances from one point to all other points. The smaller the sum, the shorter the path from this point to all other points, which means that the point is closer to all other points. The levels of these three parameters represented the topological importance of the nodes in the network, they reflected the role and influence of the corresponding nodes in the whole network and importance of the nodes was positively correlated with the output value of the network. According to relevant literature reports, the target showing two-fold the median value was selected for $\mathrm{DC}\left[{ }^{26]}\right.$, and the target with the median value for $\mathrm{BC}$ and $\mathrm{CC}\left[{ }^{27]}\right.$ was selected to obtain more accurate core targets. Then, according to the miRNA-mRNA interaction network obtained above, the hub miRNAs corresponding to hub mRNAs is obtained.

\section{Results}

The purpose of this study is to investigate, evaluate, and summarize evidence of nerve-immunity-related miRNA in the plasma of MMD patients in order to determine useful biomarkers, new therapeutic targets, and prognosis evaluation methodologies in MDD nerve-immunity interaction therapy. We predict miRNA of TFs, route Hub miRNAs and mRNAs, and build a miRNA-mRNA network and a PPI network. 


\subsection{Identification of DE-miRNAs and DE-mRNAs.}

The combined data set of 42 healthy people and 88 MMD patients revealed 34 DE-miRNAs (20 upregulated and 14 down-regulated). Following the screening with $P<0.05$ and $\log 2$ (fold change) $\geq 2$. To visualize the DE-miRNAs, a volcano map and a heat map are constructed (Figure 4). The DE-mRNAs of 80 MMD patients and 175 healthy controls were analysed. A total of $386 \mathrm{DE}-\mathrm{mRNAs}$ were identified after screening with $\mathrm{P}<0.05$ and $\log 2$ (fold change) $\geq 2$ (181 up-regulated and 205 down-regulated). To visualize the DE-mRNAs, the volcano map and heat map are utilized (Figure 5). The top 20 miRNAs and mRNAs exhibit differential expression (Table.1A,1B).

Table 1A. The table shows the information of Top 20 differentially expressed miRNAs.

\begin{tabular}{|llll|}
\hline id & logFC & adj.P.Val & Regulated \\
\hline hsa-miR-574-5p & -110.9542898 & $1.49 \mathrm{E}-36$ & down \\
\hline hsa-miR-940 & 39.47752153 & $2.16 \mathrm{E}-06$ & up \\
\hline hsa-miR-486-5p & -38.71374032 & $1.32 \mathrm{E}-14$ & down \\
\hline hsa-miR-1225-5p & -37.92807878 & $4.51 \mathrm{E}-19$ & down \\
\hline hsa-miR-1207-3p & 18.78576063 & $3.88 \mathrm{E}-06$ & up \\
\hline hsa-miR-1207-5p & -17.826831 & $1.07 \mathrm{E}-09$ & down \\
\hline hsa-miR-137 & 13.40197958 & 0.012279941 & up \\
\hline hsa-miR-320c & -12.34701801 & 0.007034566 & down \\
\hline hsa-miR-568 & 12.06373429 & 0.030023 & up \\
\hline hsa-miR-595 & -10.80147681 & $2.64 \mathrm{E}-17$ & down \\
\hline hsa-miR-1825 & 10.66823191 & 0.005454226 & up \\
\hline hsa-miR-1290 & 9.531297239 & 0.016235855 & up \\
\hline hsa-miR-450b-3p & 8.988693552 & 0.002201271 & up \\
\hline hsa-miR-621 & 7.682599653 & 0.035136805 & up \\
\hline hsa-miR-1246 & -6.396313788 & 0.000500662 & down \\
\hline hsa-miR-630 & -6.024802257 & $8.95 \mathrm{E}-09$ & down \\
\hline hsa-miR-875-3p & 4.4232763 & 0.012241154 & up \\
\hline hsa-miR-325 & 4.178569237 & $1.23 \mathrm{E}-05$ & up \\
\hline hsa-miR-127-3p & 4.163199662 & 0.015960629 & up \\
\hline hsa-miR-483-5p & -4.110638734 & 0.00430359 & down \\
\hline
\end{tabular}


Table 1B. The table shows the information of Top 20 differentially expressed mRNAs.

\begin{tabular}{|llll|}
\hline id & logFC & adj.P.Val & Regulated \\
\hline P2RY8 & -3.420464032 & $5.27 \mathrm{E}-11$ & down \\
\hline C16orf54 & -3.414050815 & $5.07 \mathrm{E}-10$ & down \\
\hline PPBP & -3.288699839 & $2.10 \mathrm{E}-10$ & down \\
\hline PLP1 & 3.192684606 & $4.51 \mathrm{E}-07$ & up \\
\hline PLAC8 & -3.1541482 & $3.41 \mathrm{E}-11$ & down \\
\hline IL2RG & -3.145809707 & $7.92 \mathrm{E}-12$ & down \\
\hline S100A9 & -3.127308745 & $1.67 \mathrm{E}-09$ & down \\
\hline CXCR2 & -3.112538855 & $1.08 \mathrm{E}-08$ & down \\
\hline MPEG1 & -3.112269482 & $1.12 \mathrm{E}-11$ & down \\
\hline CSF2RB & -3.102043325 & $7.06 \mathrm{E}-12$ & down \\
\hline CMTM2 & -3.069217774 & $7.29 \mathrm{E}-12$ & down \\
\hline CSTA & -3.061488684 & $2.90 \mathrm{E}-11$ & down \\
\hline C5AR1 & -3.014644308 & $1.18 \mathrm{E}-09$ & down \\
\hline NCF2 & -3.012015175 & $1.70 \mathrm{E}-08$ & down \\
\hline SELL & -2.992395615 & $1.10 \mathrm{E}-10$ & down \\
\hline S100A8 & -2.96753421 & $3.42 \mathrm{E}-08$ & down \\
\hline IL7R & -2.949694197 & $4.84 \mathrm{E}-11$ & down \\
\hline EOMES & -2.941185679 & $4.82 \mathrm{E}-11$ & down \\
\hline PLBD1 & -2.936378649 & $7.59 \mathrm{E}-12$ & down \\
\hline FCGR3B & -2.910908361 & $2.51 \mathrm{E}-09$ & down \\
\hline
\end{tabular}

\subsection{Predicting TF and target genes of miRNAs.}

SP1, SP4, KLF7EGR1, HNF4A, POU2F1, GABPA, ETS1, CTCF, and RREB1 are the top ten TF in DE-miRNAs (Figure 6-A). SP1, SP4, KLF7, EGR1, HNF4A, CTCF, POU2F1, NFYA, GABPA, and RREB1 were the top ten TFs of up-regulated DE-miRNAs (Figure 6-B). SP1, SP4, KLF7, EGR1, HNF4A, POU2F1, GABPA, ETS1, MEF2A, and NFIC are the top ten TFs of down-regulated DE-miRNAs. (Figure 6-C) successfully predicted the target mRNA for 578 up-regulated and 635 down-regulated DE-miRNAs. See (Appendix Table 1) for TF analysis and miRNA target genes (Appendix Table 2). 


\subsection{Functional annotation of DE-miRNAs}

FunRich program discovered 59 biological processes (BP), 267 cellular composition (CC), and 116 molecular functions (MF) using GO enrichment analysis of DE-mRNAs (Figure 7). It is essentially connected to Molecular function, Transcription activity, and DNA-RNA-Protein activity in the MF, Molecular function uncertain, Transcription factor activity, Transcription regulator activity, DNA-RNA binding, Protein serine/threonine kinase activity, and so on. It is primarily connected to signal and cell communication in the BP, such as signal transduction, cell communication, biological process unknown, and regulation of nucleobase, nucleoside, nucleotide, and nucleic acid metabolism. It is mainly associated with Cytoplasm, Nucleus, Plasma membrane, Exosomes, and Lysosomes in the CC (Appendix Table 3-5).

\subsection{Identifying the intersection mRNAs.}

The 34 DE-miRNAs (20 up-regulated and 14 down-regulated) and 578 up-regulated and 635 downregulated target mRNAs effectively predicted were compared to the $386 \mathrm{DE}-\mathrm{mRNAs}$ (181 up-regulated and 205 down-regulated) acquired from GEO data screening. The VENN map (Figure 8) was constructed, which included 17 overlapping DE-mRNAs. Crossover genes are represented in (Table 2).

Table 2. The table shows the information of intersection genes. 


\begin{tabular}{|lllll|}
\hline miRNA & mRNA & Target & mirnaLogFC & mrnaLogFC \\
\hline hsa-miR-206 & GJA1 & target & -3.251413682 & 2.682957112 \\
\hline hsa-miR-206 & MAL2 & target & -3.251413682 & 2.253644367 \\
\hline hsa-miR-206 & PCDH17 & target & -3.251413682 & 2.035934044 \\
\hline hsa-miR-206 & SNAP25 & target & -3.251413682 & 2.533742062 \\
\hline hsa-miR-206 & SOX9 & target & -3.251413682 & 2.070698257 \\
\hline hsa-miR-320c & GNA11 & target & -12.34701801 & 2.155037624 \\
\hline hsa-miR-320c & LMO3 & target & -12.34701801 & 2.604451601 \\
\hline hsa-miR-320c & TUSC3 & target & -12.34701801 & 2.034421167 \\
\hline hsa-miR-338-3p & CACNA2D1 & target & -2.295135247 & 2.092690723 \\
\hline hsa-miR-338-3p & CHL1 & target & -2.295135247 & 2.234787943 \\
\hline hsa-miR-338-3p & KCND2 & target & -2.295135247 & 2.141220267 \\
\hline hsa-miR-338-3p & MYT1L & target & -2.295135247 & 2.069355937 \\
\hline hsa-miR-338-3p & NOVA1 & target & -2.295135247 & 2.202273914 \\
\hline hsa-miR-296-5p & RAB37 & target & 2.171635035 & -2.470323316 \\
\hline hsa-miR-137 & BAZ1A & target & 13.40197958 & -2.100731 \\
\hline hsa-miR-137 & RNASE6 & target & 13.40197958 & -2.659559722 \\
\hline hsa-miR-137 & SASH3 & target & 13.40197958 & -2.661132519 \\
\hline hsa-miR-127-3p & RAB37 & target & 4.163199662 & -2.470323316 \\
\hline
\end{tabular}

\subsection{Construction of miRNA-mRNA network and PPI network}

Because miRNA and mRNA have a negative regulatory interaction, the previously collected intersecting DE-mRNAs were employed in Cytoscape3.7.2 to construct a miRNA-mRNA regulatory network. The network contains 6 miRNA and 17 mRNA (Figure 9). Table 1 indicates the comparable expression of miRNA-mRNA in MMD.

\subsection{Construction of PPI network and Screening of hub mRNAs and miRNAs.}

To obtain the PPI network, the common differentially expressed genes from the two data sets are introduced in the String database, and the unconnected targets are deleted. Cytoscape3.7.2 BisoGenet revealed that the network had 369 nodes and 2485 edges. The CytoNCA plug-in is then used to further evaluate the network's core targets, depending on the features of the network architecture. Depending on the $\mathrm{DC}$, the target with twice the median value is chosen, whereas $\mathrm{BC}$ and $\mathrm{CC}$ to choose the target of the 
median value. Following screening, five hub mRNAs and their corresponding hub miRNAs (Appendix Table 6-7) are defined, and the node transmission information and transmission efficiency are chosen as the "primary target" (Figure 10) for further investigation. It can be pointed out that the core genes include KCND2, MYT1L, GJA1, CHL1, and SNAP25 (Table 3).

Table 3. Information on 5 core targets.

\begin{tabular}{|c|c|c|c|c|c|}
\hline SUID & $\begin{array}{l}\text { Gene } \\
\text { symbol }\end{array}$ & Protein name & Betweenness & Closeness & Degree \\
\hline 77 & KCND2 & $\begin{array}{l}\text { Potassium voltage-gated channel } \\
\text { subfamily D member } 2\end{array}$ & 0.666666667 & 1 & 4 \\
\hline 83 & $\mathrm{CHL} 1$ & $\begin{array}{l}\text { Neural cell adhesion molecule L1-like } \\
\text { protein }\end{array}$ & 0.666666667 & 1 & 4 \\
\hline 73 & SNAP25 & Synaptosomal-associated protein 25 & 0.666666667 & 1 & 4 \\
\hline 92 & MYT1L & $\begin{array}{l}\text { Myelin transcription factor 1-like } \\
\text { protein }\end{array}$ & 0 & 0.8 & 3 \\
\hline 75 & GJA1 & Gap junction alpha- 1 protein & 0 & 0.8 & 3 \\
\hline
\end{tabular}

\subsection{Go and KEGG Enrichment analysis of miRNA-mRNA regulatory network.}

470 biological process (BP), 83 cellular composition (CC) and 84 molecular function (MF) (Figure 11) were identified by $\mathrm{GO}$ enrichment analysis of common DEMs by R4.1.0 software. In terms of molecular function, mainly including channel activity (GO:0015267), passive transmembrane transporter activity (GO:0022803), immune receptor activity (G0:0140375), nucleoside binding (GO:0001882). In the biological process, mainly including neutrophil activation (GO:0042119), neutrophil activation involved in immune response (G0:0002283), neutrophil degranulation (G0:0043312), neutrophil mediated immunity (G0:0002446). In the cellular composition, mainly including presynapse (G0:0098793), external side of plasma membrane (G0:0009897), secretory granule membrane (G0:0030667), synaptic membrane (GO:0097060) (Appendix Table 8-10). We selected the first 20 feature-rich processes to draw clusters and circles, as showed in (Figure 9). In addition, we identified the main signal pathways involved in the occurrence and development of MMD in KEGG enrichment analysis, and screened out the first 20 signal pathways related to MMD and significantly enriched. Including Cytokine-cytokine receptor interaction (hsa04060), Chemokine signaling pathway (hsa04062), Phagosome (hsa04145), Tuberculosis (hsa05152), Viral protein interaction with cytokine and cytokine receptor (hsa04061), Neutrophil extracellular trap formation (hsa04613) (Appendix Table 11). We select the first 20 principal signal pathways to draw cluster diagrams and circles, such as figure (Figure 12).

\section{Discussion}

The incidence of MMD is increasing year by year as a result of the pressures of job and life. Patients with MMD not only suffer from the condition, but they also make an unbelievable load on their family and 
society as a whole ${ }^{28]}$. Modern medicine examines the pathogenic basis of MMD to be a disrupted metabolic process of the neuro-endocrine-immune network consists of the neurological system, hypothalamus-pituitary-adrenal axis (HPA axis), and immune system[29-][a30a]. Depending on studies, nerve-immunity network malfunction causes excessive secretion of inflammatory components and induces glucocorticoid secretion, which mediates the onset of MMD[11]. The increased expression of proinflammatory factors such as TNF-, IL-6, and IL-1 in the serum, cerebrospinal fluid, and hippocampus of MMD patients and animal models forms a negative feedback regulation of the anti-inflammatory factor $\mathrm{IL}-10$, resulting in neuronal inflammation and neuronal damage in the central nervous system[ $\left.{ }^{32}\right][$ a33a].

At present, scholars place great importance on nerve-immunity interactive therapy MDD. Commonly used clinical treatments such as escitalopram, fluoxetine, acupuncture and Chinese materia medica are related to nerve-immunity interactive therapy. It has been found that common antidepressants such as escitalopram and fluoxetine can enhance central function by enhancing central serotonergic nerve activity. It can also effectively regulate the levels of serum brain-derived neurotrophic factors and inflammatory factors in patients $\left[{ }^{34-}\left[{ }_{a} 35 a\right]\right.$. Acupuncture can increase the content of serum $5-\mathrm{HT}$, upregulate the content of anti-inflammatory cytokine IL-10, and reduce the content of proinflammatory cytokine IL-6 and TNF-a[ $\left.\left.{ }^{36-1}\right]_{a} 37 a\right]$. Chinese materia medica such as Rhizoma Cyperi, Rhizoma Coptidis, Cinnamomum and Radix Paeoniae Alba can improve the growth and development of neurons by mediating neurotrophic factors and other signal pathways, resist the neuronal damage caused by MMD, and regulate the signal transduction pathways such as NLR, ErbB and chemokines to regulate inflammatory response and immune response, resulting in antidepressant effects[38-][a39a[i]][a40a[ii]] [a41a]. It has been reported that miRNAs plays a major role in the occurrence and development of MMD, but the interaction between miRNAs and mRNA is not clear. Therefore, it is of profound significance to study MMD at the molecular level and to find the mechanism of nerve-immunity interactive therapy of MDD, so as to provide reference ideas for future basic research and clinical application.

We discovered 17 important Hub genes by the development of a miRNA-mRNA network, and after CytoNCA topology, we discovered 5 Hub DE-MRNAs: KCND2, MYT1L, GJA1, CHL1, and SNAP25, all of which are up-regulated genes. The comparable miRNAs are hsa-miR-206 and hsa-miR-338-3p, which are both down-regulated in MMD. Although five pairs of regulatory relationships between miRNA and mRNA have not previously been reported, this study validates hsa-miR-206 with GJA1, SNAP25, hsa-miR-338-3p with KCND2, MYT1L through the miRDB database (http://mirdb.org/), and hsa-miR-338-3p with CHL1 through the TargetScan database (http://www.targetscan.org/vert_71/), and predicts the possibility of interaction between five pairs of miRNA-mRNA. It provides a theoretical foundation for additional experimental validation of the coupling of these miRNA and mRNA.

MiRNAs are tiny non-coding RNAs that directly regulate more than $30 \%$ of the genes in cells by inhibiting the degradation or translation of target mRNAs [2-][a43a]. It is linked to nearly every fundamental biological function, including MMD and anxiety disorders [ $\left.{ }^{44}\right][\mathrm{a} 45 \mathrm{a}] . \mathrm{It}$ is understood that changes in miRNA expression are linked to the pathophysiology of numerous neurodegenerative disorders[ ${ }^{46]}$. MiR- 
206, a member of the muscle-specific miR-1 family, was first considered to govern skeletal muscle embryonic development ${ }^{47]}$. MiR-338-3p was first thought to be a cancer-related miRNA, but it was later found to be involved in neuronal regulation, including the Parkinson's disease pathway ${ }^{48]}$. Neurological aspect: Brain-derived neurotrophic factor (BDNF) participates in neuroplasticity and protection throughout brain development, as well as playing an important role in emotion-related brain activities [ $\left.{ }^{49}\right][\mathrm{a} 50 \mathrm{a}]$. MiR206 has the capacity to suppress the production of BDNF following transcription, which is vital in the regulation and participation in $\mathrm{MMD}\left[{ }^{51]}\right.$.It was also discovered that using miR-206 antagonists could attenuate stress-induced aggressive behavior while increasing BDNF expression in SI mice. Up-regulation of miR-206 has been demonstrated to impair neuron function and result in poor adaptive behavior[25]. MiR-338 can increase myelination in the nervous system, and over-expression of miR-338 can boost oligodendrocyte differentiation and inhibit myelin negative regulators Sox 6 and Hes5, hence encouraging myelin formation[53-I [a54a]. MiR-338-5p has been found to safeguard the cognitive function of transgenic APP/PS1 mice by lowering neuronal death[55]. Other research has shown that silencing miR-338-5p can result in neuronal polarity loss and a considerable decrease in the number of neurons. Thus, activating miR-338 can be, to some extent, influences the emotional function of the brain [ ${ }^{56]}$. Immune aspects: MiR206 is also linked to the TNF signaling pathway [ ${ }^{57]}$. The activated TNF pathway mediated by miR-206 can, in turn, drive NF-KB to enter the nucleus and boost the creation and release of inflammatory mediators such as TNF-a, IL-8, IL-6, and others to be involved in inflammatory and immunological processes ${ }^{58]}$. TNF inflammatory cytokines, which are plentiful in tumor microenvironments, can promote tumor growth, disrupt cell proliferation and death, and impair the innate immune response to cancer cells[59]. MiR-338-3p is also related to the TNF signaling pathway. TNF-a may cause inflammation by activating the NF-KB/MAPK signaling pathway and lowering the expression of miR-338-

$3 p\left[{ }^{60]}\right.$. Overexpression of miR-338-3p has been shown to block the ERK/p38MAPK signaling pathway and reduce the expression of pro-inflammatory genes VCAM-1 and ICAM-1RNA[61].

KCND2 is a Potassium Voltage-Gated Channel Subfamily D protein coding gene that can encode a type A potassium channel protein that is important during the repolarization stage of an action potential[ ${ }^{62]}$. By controlling potassium transport across the excitatory membrane in the brain, voltage-gated potassium channels regulate dendritic A-type current I (SA) in brain neurons ${ }^{[3]}$. By controlling potassium transport across the excitatory membrane in the brain, voltage-gated potassium channels regulate dendritic A-type current I (SA) in brain neurons ${ }^{[64]}$. Myt1L (myelin transcription factor 1 like) is a fellow of the Myt/neural zinc finger (NZF) family that encodes a neural transcription factor with six zinc fingers. Its expression has so far only been detected in neural tissue[ ${ }^{65]}$. This gene is important in order to neural differentiation, and mutations in it have been linked to autism spectrum disorder and an autosomal dominant form of cognitive dysfunction [ ${ }^{66}$. Mutations in Myt1 L in chromosomal band 2p25.3 have been linked to intellectual disability, while Myt1L repetition has been linked to schizophrenia and MMD[67]. GJA-1 (Gap junction alpha-1 protein) expression had proven to be down regulated in MDD patients, as was the $\mathrm{Cx} 43$ mRNA that encoded it[ ${ }^{68]}$. CHL1 (Cell Adhesion Molecule L1 Like) is a cell adhesion molecule that belongs to the L1 family. This gene's deletion and repetition have been associated with a variety of neurological 
illnesses including autism, Parkinson's disease, and mental impairment $\left[{ }^{[69]}\right.$. The study revealed that the level of serum $\mathrm{CHL} 1$ in patients with MMD declined dramatically and was inversely proportional to the severity of the disease, implying that CHL1 may be involved in the pathogenesis of MMD and immune cell dysfunction [ ${ }^{70}$. SNAP-25 has been associated with the intensity of depressive symptoms in patients with MMD as judged by the Beck MMD Rating Scale in numerous studies[11-J[a72a[iii]][a73a]. It can be seen that both the hub miRNA and the mRNA generated by establishing a miRNA-mRNA network plays a role in the occurrence and progression of MMD via nerve-immunity interaction.

To examine the molecular mechanism of MDD, we investigated the key targets using GO and KEGG enrichment. The core targets added value in immunological and neural aspects in BP, CC, and MF, according to GO and KEGG enrichment analysis. Presynapse, synaptic membrane, neuronal cell body, synaptic connections between neurons, and voltage-gated sodium channel complex were among the 83 $\mathrm{CC}$ that were found to be enriched. There were 84 enriched elements in the MF, most of which were immune receptor activity, neurotransmitter receptor activity, chemokine binding, etc. There were 470 enriched elements in the BP, most of which were connected with neutrophil, $\mathrm{T}$, and B cell activation, activation of surface receptors and signal pathways of immune response, and modulation of chemical synaptic transmission, among other things. In addition, 39 MMD-related pathways were determined and evaluated, including immune and neuroregulation pathways.

Among the signaling pathways investigated in this work. The signal pathways listed below being associated with immunological and neurological control. Specifically, the B and T cell receptor signal pathways, the chemokine signal route, the JAK-STAT signal pathway, Th1 and Th2 cell differentiation, and Th17 cell differentiation. Th17, Th1, and Th2 CD4+T lymphocyte subsets of the human immune system serve essential roles in promoting inflammation and immunosuppression, respectively, and contribute in immune system homeostasis while supporting B cell activation and having a role in humoral immunity[ ${ }^{74-J}$ a75a]. $\otimes$ TTh17 cell differentiation: Th17 plays a role in the pathophysiology of MDD by increasing the generation of autoantibodies and the proclivity for autoimmunity ${ }^{76]}$. Furthermore, Th17 and Treg are connected in differentiation and function, and they are frequently in a state of dynamic equilibrium. If the proportion of them is no longer part balanced, it will result in aberrant immunological responses such as inflammatory, tumor, and autoimmune reactions. [77]. Th17 had proven to be elevated in the brains of MMD model mice, but RORt defective mice demonstrated resistance to learning helplessness. As a result, an increase in Th17 can cause MMD, whereas suppressing Th17 production or function can lessen the risk of MMD in mice[ ${ }^{78]}$. $\otimes T h 1$ and Th2 cell differentiation: Both Th1 and Th2 cells can release cytokines, which can boost their own proliferation while inhibiting each other in order to maintain a relative equilibrium. When the equilibrium is disrupted, also known as "Th1/Th2 drift," it can be expected to result in the emergence and development of a variety of disorders associated with immunological escape ${ }^{79-}\left[\left[_{0} 80 a\right] . ~ \otimes T\right.$ and B signaling pathways: In MMD patients, immunological function was compromised, T cell apoptosis increased, neutrophils rose, and total lymphocytes decreased. T cell function degradation could be due to inflammatory substances such as tumor necrosis factor alpha destroying $T$ cell function. B cells mediate humoral immunity via antibodies; 
as the number of B cells falls, so does the rate of transformation $\left[{ }^{81]}\right.$. $\otimes$ Chemokine signaling pathway: Chemokines' major purpose is to control leukocyte migration (homing) to their proper regions during inflammation and homeostasis. Some regulate the chemotaxis of immune cells during the immune surveillance process, and others recruit neutrophils to the site of infection or tissue injury for antiinfection, which serves a guiding role in the cells of the innate immune system and the adaptive immune

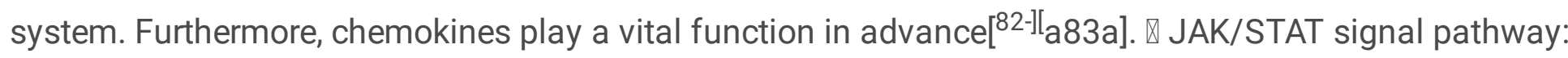
Not only gets this one of the most essential transmembrane signal transduction pathways, but it is also the most critical signal pathway triggered by inflammatory cytokines ${ }^{84]}$. Furthermore, JAK and STAT are key components of many signaling pathways that regulate cell growth, differentiation, survival, and pathogen resistance, including those involving the IL-6 (gp130) receptor family, which aids in the regulation of B cell differentiation, plasma cell production, and acute phase response[ $\left.{ }^{85}\right][$ a86a]. Neural aspect: The JAK/STAT signaling pathway is widely implicated in the proliferation and differentiation of NSC during central nervous system development, and it plays a key role in regulating hippocampal nerve remodeling ${ }^{87]}$. A member of its family, the JAK2/STAT3 signal pathway, is involved in neuronal death and nervous reorganization in ischemic encephalopathy $\left[{ }^{[8]}\right.$. Numerous studies have revealed that TNF-a causes JAK/STAT signal pathway activation via the protein-gp130 on the cell membrane's surface, and so contributes to the control of central nervous system degenerative alterations [ ${ }^{89-1[a 90 a]}$. In addition, the JAK/STAT signaling pathway can influence neuron reorganization via inflammatory cytokines, brainderived neurotrophic factors, neurotransmitters, etc ${ }^{91-1[}$ a92a[iv]][a93a]. As a consequence, medications and other treatment interventions can suppress neuronal death and promote nerve reorganization by altering the JAK/STAT signal pathway's associated molecules.

Because of the blood-brain barrier, the central nervous system is commonly considered to be an immunological zone, according to conventional wisdom. However, as more study is undertaken, this concept is gradually shifting[94-][a95a]. Chemokines, which include Th17, Th1, and Th2, are key chemicals in the immunological and neurological systems. They can coordinate this migration of $\mathrm{T}$ and $\mathrm{B}$ cells to inflammatory areas, promote $\mathrm{T}$ cell activity and help $\mathrm{B}$ cell activation, and play a function in regulating immunity-inflammation in the immune system[ ${ }^{96}$. Following psychological stress, activated T cells can get into the central nervous system in a variety of ways, reduce inflammation, and support neuronal integrity via cellular immunity, so modifying depressed behavior[ ${ }^{[97-[}[\mathrm{a} 98 \mathrm{a}]$. Furthermore, the JAK/STAT signaling system influences brain remodeling by activating inflammatory cytokines and altering the metabolism of the neuro-endocrine-immune network.

Depending on the Integrated Analysis of miRNA-mRNA Regulatory Networks, miR-338-3p and miR-206 can coordinate the regulation of the neuro-endocrine-immune network by regulating chemokines such as Th17, Th1 and Th2, immune cells such as T/B and the JAK/STAT signaling pathway, among other things. Realize bi-directional regulation of the HPA axis, which can normalize nerve conduction, minimize immuno-inflammatory responses, and restore normal neural, endocrine, and immunological function, and so play a therapeutic role in MMD. To research useful biomarkers and treatment targets from the perspective of MMD and the host neuro-endocrine-immune network in order to regulate the level of 
immune inflammatory cytokines in the microenvironment and balance neuro-endocrine-immune homeostasis at the molecular level. It is critical to improve the critical mechanism of neuro-endocrineimmune imbalance in MMD patients.

[[i]] Zhang JingHua,Yang HuiZeng,Su Hao,Song Jun,et al. Berberine and Ginsenoside Rb1 Ameliorate Depression-Like Behavior in Diabetic Rats.[J]. The American journal of Chinese medicine,2021:

[[ii]] Zhihuan Zhou,Shufei Fu,Yijia Liu,et al. Study of efficacy and safety of Jiaotai pill in the treatment of depression[J]. Medicine,2020,99(18):

[[iii]] Alfredo Ramos-Miguel,Kristina Gicas,Jehan Alamri,et al. Beasley,Andrew J. Dwork,J. John Mann,Gorazd Rosoklija,Fang Cai,Weihong Song,Alasdair M. Barr,William G. Honer. Reduced SNAP25 Protein Fragmentation Contributes to SNARE Complex Dysregulation in Schizophrenia Postmortem Brain[J]. Neuroscience,2019,420:

[[iv]] Hixson Kathryn M,Cogswell Meaghan,Brooks-Kayal Amy R,et al. Evidence for a non-canonical JAK/STAT signaling pathway in the synthesis of the brain's major ion channels and neurotransmitter receptors.[J]. BMC genomics,2019,20(1):

\section{Conclusions}

To summarize, the occurrence and progression of MMD are a function of multi-target, multi-pathway, multi-signal pathway, and multi-mechanism interactions, and the regulation process is synergistic and bidirectional. MiRNA, which differs from miR-206 and miR-338-3p, may activate or inhibit the B cell receptor signal pathway, $T$ cell receptor signal pathway, and chemokine signal pathway by governing the expression of KCND2, Myt1L, GJA-1, CHL1, and SNAP-25. JAK-STAT and other signal pathways regulate immune-inflammatory response, nerve remodeling, and other functions, as well as mediating the occurrence and progression of MMD. Although this study presents some theoretical foundations and research ideas for nerve-immunity interaction, it has several drawbacks. Improvement suggestions: $\mathbb{\square}$ Because the current data is derived from the GEO database, it is impossible to assess the dependability and quality of the statistical data. In the future, we will be added to the number of data sources and decrease the data offset. $\triangle$ Conduct additional scientific and clinical research to see if acupuncture, TCM, and other treatments can improve the neurological and immunological function of MMD patients by modulating the level of miRNA in the brain.

\section{Abbreviations}

\begin{tabular}{|llll|}
\hline MDD & Major depressive disorder & GEO & Gene Expression Omnibus GEO, \\
\hline GO & Gene Ontology & MiRNAs & MicroRNAs MiRNAs) \\
\hline TCM & traditional Chinese medicine & BP & Biological processes \\
\hline MF & molecular functions & CC & cellular components \\
\hline KEGG & Kyoto Encyclopedia of Genes and Genomes & DEGs & differentially expressed genes \\
\hline
\end{tabular}




\section{Declarations}

\section{Data availability}

1. All the data can be obtained from the open source website we provide, and the conclusion can be drawn through he analysis of the relevant software.

2. The [miRNA] data used to support the findings of this study have been deposited in the [GEO] repository (https://www.ncbi.nlm.nih.gov/geo/).

3. The [mRNA] data used to support the findings of this study have been deposited in the [GEO] repository (https://www.ncbi.nlm.nih.gov/geo/)

4. The [Integrated Analysis of miRNA-mRNA Regulatory Networks] data used to support the findings of this study are included within the supplementary information file(s).

\section{Ethics approval and consent to participation}

This manuscript is not a clinical trial, hence the ethics approval and consent to participation is not applicable.

\section{Consent for publication}

All authors have read and approved this manuscript to be considered for publication.

\section{Competing interests}

The authors declare no competing financial interests.

\section{Acknowledgements}

Thanks to professor Huang for his strict guidance on this paper, and thanks to Miss Huang and Miss Cai of support for this paper. Thanks to reviewers and editors for their sincere comments.

\section{Fund}

1. National Natural Science Foundation of China (NSFC), 82160938, "The effect of TGF- $\beta$ 1-mediated ERK and Smad signaling pathway on the anti-renal interstitial fibrosis mechanism of drug-cake moxibustion"; 2. Health And Health Commission of Yunnan Province 2020 High-level TCM Reserve Talents (Acupuncture treatment of chronic kidney disease) Incubation project (Yunwei TCM Development Development [2021] No. 1) 3. The second round of construction project of The National Traditional Chinese Medicine School Heritage Studio of the State Administration of Traditional Chinese Medicine (National Traditional Chinese Medicine Teaching Letter [2019] 62); 4. Yunnan Provincial Health Commission "2020 High-level Traditional Chinese Medicine Talents Training Target" project (Yunnan Traditional Chinese Medicine Development (2021) No. 1); 5. Yunnan Provincial Department of Science and Technology-Joint Special General Project of Traditional 
Chinese Medicine (No.2019FF002-022); 6. Social Science Project of Yunnan University of Traditional Chinese Medicine (No. [2020] -SkyB-00016).

\section{Author Contributions}

Zixuan Wu drafted and revised the manuscript. Xuyan Huang and Minjie Cai are in charge of data collection. Peidong Huang conceived and designed this article, in charge of syntax modification and revise of the manuscript. Zunhui Guan revised the manuscript. All the authors have read and agreed to the final version manuscript.

\section{References}

1 Ding Yuqiang,Dai Jinxia. Advance in Stress for Depressive Disorder.[J]. Advances in experimental medicine and biology,2019,1180:

2 David A. Casey. Depression in Older Adults: A Treatable Medical Condition[J]. Primary Care: Clinics in Office Practice,2017,44(3):

3 Scott M. Thompson,Angy J. Kallarackal,Mark D. Kvarta,Adam M. Van Dyke,Tara A. LeGates,Xiang Cai. An excitatory synapse hypothesis of depression[J]. Trends in Neurosciences,2015,38(5):

4 Marie Ng,Tom Fleming,Margaret Robinson,et al. Global, regional, and national prevalence of overweight and obesity in children and adults during 1980-2013: a systematic analysis for the Global Burden of Disease Study 2013[J]. The Lancet,2014,384(9945):

5 Xie Weijie,Meng Xiangbao,Zhai Yadong,et al. Panax Notoginseng Saponins: A Review of Its Mechanisms of Antidepressant or Anxiolytic Effects and Network Analysis on Phytochemistry and Pharmacology.[J]. Molecules (Basel, Switzerland),2018,23(4):

6 Dionisie Vlad,Filip Gabriela Adriana,Manea Mihnea Costin,et al. Neutrophil-to-Lymphocyte Ratio, a Novel Inflammatory Marker, as a Predictor of Bipolar Type in Depressed Patients: A Quest for Biological Markers.[J]. Journal of clinical medicine,2021,10(9):

7 Yang C R,Ning L,Zhou F H,et al. Downregulation of Adhesion Molecule CHL1 in B Cells but Not T Cells of Patients with Major Depression and in the Brain of Mice with Chronic Stress.[J]. Neurotoxicity research,2020,38(4):

8 Bhattacharya Anindya,Drevets Wayne C. Role of Neuro-Immunological Factors in the Pathophysiology of Mood Disorders: Implications for Novel Therapeutics for Treatment Resistant Depression.[J]. Current topics in behavioral neurosciences,2017,31:

9 Chamberlain Samuel R,Cavanagh Jonathan,de Boer Peter,et al. Treatment-resistant depression and peripheral C-reactive protein.[J]. The British journal of psychiatry : the journal of mental science,2019,214(1): 
10 Vasileva Liliya V,Ivanovska Mariya V,Murdjeva Marianna A,et al. Immunoregulatory natural compounds in stress-induced depression: An alternative or an adjunct to conventional antidepressant therapy?[J]. Food and chemical toxicology : an international journal published for the British Industrial Biological Research Association,2019:

11 Mike Armour,Caroline A. Smith,Li-Qiong Wang,et al. Acupuncture for Depression: A Systematic Review and Meta-Analysis[J]. Journal of Clinical Medicine,2019,8(8):

12 Xiao Li,Xue-Mei Qin,Jun-Sheng Tian,et al. Integrated network pharmacology and metabolomics to dissect the combination mechanisms of Bupleurum chinense DC -Paeonia lactiflora Pall herb pair for treating depression[J]. Journal of Ethnopharmacology,2021,264:

13 Julie Cazareth,Alice Guyon,Catherine Heurteaux,et al. Molecular and cellular neuroinflammatory status of mouse brain after systemic lipopolysaccharide challenge: importance of CCR2/CCL2 signaling[J]. BioMed Central,2014,11(1):

14 Wang Xiaoyu,Chen Shuangshuang,Ni Jingshu,et al. miRNA-3473b contributes to neuroinflammation following cerebral ischemia.[J]. Cell death \& disease,2018,9(1):

15 Xiaolin Yang,Qinyan Yang,Xiaobin Wang,et al. MicroRNA Expression Profile and Functional Analysis Reveal that miR-206 is a Critical Novel Gene for the Expression of BDNF Induced by Ketamine[J]. NeuroMolecular Medicine,2014,16(3):

16 Fiori Laura M,Kos Aron,Lin Rixing,et al. miR-323a regulates ERBB4 and is involved in depression.[J]. Molecular psychiatry,2020:

17 Lauren Allen,Yogesh Dwivedi. MicroRNA mediators of early life stress vulnerability to depression and suicidal behavior[J]. Molecular Psychiatry,2020,25(2):

18 Elisabetta Maffioletti,Annamaria Cattaneo,Gianluca Rosso,et al. Peripheral whole blood microRNA alterations in major depression and bipolar disorder[J]. Journal of Affective Disorders,2016,200:

19 Tao Ziqi,Shi Aimin,Li Rui,et al. Microarray bioinformatics in cancer- a review.[J]. Journal of B.U.ON. : official journal of the Balkan Union of Oncology,2017,22(4):

20 Fonseka Pamali,Pathan Mohashin,Chitti Sai V,et al. FunRich enables enrichment analysis of OMICs datasets[J]. Journal of Molecular Biology,2020(prepublish):

21 Paul Shannon,Andrew Markiel,Owen Ozier,et al. Cytoscape: A Software Environment for Integrated Models of Biomolecular Interaction Networks[J]. Cold Spring Harbor Laboratory Press,2003,13(11):

22 Andrea F,Damian S,Sune F,et al. STRING v9.1: protein-protein interaction networks, with increased coverage andintegration[J]. Nucleic Acids Res,2013,41(Database issue):D808-D815. 
23 Patrycja Vasilyev Missiuro,Kesheng Liu,Lihua Zou,et al. Information Flow Analysis of Interactome Networks[J]. PLOS Computational Biology,2009,5(4):

24 Karthik Raman,Nandita Damaraju,Govind Krishna Joshi. The organisational structure of protein networks: revisiting the centrality-lethality hypothesis[J]. Systems and Synthetic Biology,2014,8(1):

25 Yu Tang,Min Li,Jianxin Wang,et al. CytoNCA: A cytoscape plugin for centrality analysis and evaluation of protein interaction networks[J]. BioSystems,2015,127:

26 Song Wenjie,Ni Shenglou,Fu Yanling,et al. Uncovering the mechanism of Maxing Ganshi Decoction on asthma from a systematic perspective: A network pharmacology study.[J]. Scientific reports,2018,8(1):

27 Wan Yuxiang,Xu Lin,Liu Zeyu,et al. Utilising network pharmacology to explore the underlying mechanism of Wumei Pill in treating pancreatic neoplasms.[J]. BMC complementary and alternative medicine,2019,19(1):

28 Alize J Ferrari,Emily Stockings,Jon-Paul Khoo,et al. The prevalence and burden of bipolar disorder: findings from the Global Burden of Disease Study 2013[J]. Bipolar Disorders, 2016,18(5):

29 Pan Changqie,Wu Jianhua,Zheng Siting,et al. Depression accelerates gastric cancer invasion and metastasis by inducing a neuroendocrine phenotype via the catecholamine/ $\beta 2$-AR/MACC1 axis.[J]. Cancer communications (London, England),2021:

30 Gold Philip W,Wong MaLi. Re-assessing the catecholamine hypothesis of depression: the case of melancholic depression.[J]. Molecular psychiatry,2021:

31 Pan Xu,Kezhu Wang,Cong Lu,et al. Effects of the chronic restraint stress induced depression on reward-related learning in rats[J]. Behavioural Brain Research,2017,321:

32 Jia Jia Liu,Ya Bin Wei,Rebecca Strawbridge,et al. Gadad,Madhukar H. Trivedi,John R. Kelsoe,Lin Lu. Peripheral cytokine levels and response to antidepressant treatment in depression: a systematic review and meta-analysis[J]. Molecular Psychiatry,2020,25(2):

33 Dang Ruili,Zhou Xueyuan,Tang Mimi,et al. Fish oil supplementation attenuates neuroinflammation and alleviates depressive-like behavior in rats submitted to repeated lipopolysaccharide.[J]. European journal of nutrition,2018,57(3):

34 Dionisie Vlad,Ciobanu Adela Magdalena,Toma Vlad Alexandru,et al. Escitalopram Targets Oxidative Stress, Caspase-3, BDNF and MeCP2 in the Hippocampus and Frontal Cortex of a Rat Model of Depression Induced by Chronic Unpredictable Mild Stress.[J]. International journal of molecular sciences,2021,22(14):

35 Ju Xuan,Wang Shengdong,Yan Pan,et al. Rapid Eye Movement Sleep Deprivation Combined With Fluoxetine Protects Against Depression-Induced Damage and Apoptosis in Rat Hippocampi via A1 
Adenosine Receptor [J]. Frontiers in Psychiatry,2021:

36 Hsiang-Chun Lai,Qwang-Yuen Chang,Ching-Liang Hsieh,et al. Signal Transduction Pathways of Acupuncture for Treating Some Nervous System Diseases[J]. Evidence-Based Complementary and Alternative Medicine,2019,2019:

37 Jia Jia Liu,Ya Bin Wei,Rebecca Strawbridge,et al. Peripheral cytokine levels and response to antidepressant treatment in depression: a systematic review and meta-analysis[J]. Molecular Psychiatry,2020,25(2):

38 Hong-mei Jia,Zhong-mei Zou. Antidepressant effect evaluation of the ethanolic extract from the roots of Cyperus rotundus L. on cell membrane chromatography and different depression models[J]. European Journal of Integrative Medicine,2014,6(6):

39 Zhang JingHua,Yang HuiZeng,Su Hao,Song Jun,et al. Berberine and Ginsenoside Rb1 Ameliorate Depression-Like Behavior in Diabetic Rats.[J]. The American journal of Chinese medicine,2021:

40 Zhihuan Zhou,Shufei Fu,Yijia Liu,et al. Study of efficacy and safety of Jiaotai pill in the treatment of depression[J]. Medicine,2020,99(18):

41 Chen Yanyan,Wang Wenran,Fu Xin,et al. Investigation of the antidepressant mechanism of combined Radix Bupleuri and Radix Paeoniae Alba treatment using proteomics analysis of liver tissue[J]. Journal of Chromatography B,2021(prepublish):

42 Kevin Chen,Nikolaus Rajewsky. The evolution of gene regulation by transcription factors and microRNAs[J]. Nature Reviews Genetics,2007,8(24):

43 Lewis Benjamin P,Burge Christopher B,Bartel David P. Conserved seed pairing, often flanked by adenosines, indicates that thousands of human genes are microRNA targets.[J]. Cell,2005,120(1):

44 Zhou Lisha,Zhu Yingying,Chen Wangyang,et al. Emerging role of microRNAs in major depressive disorder and its implication on diagnosis and therapeutic response[J]. Journal of Affective Disorders,2021,286:

45 Haramati Sharon,Navon Inbal,Issler Orna,et al. MicroRNA as repressors of stress-induced anxiety: the case of amygdalar miR-34.[J]. The Journal of neuroscience : the official journal of the Society for Neuroscience,2011,31(40):

46 Gruzdev S K,Yakovlev A A,Druzhkova T A,et al. The Missing Link: How Exosomes and miRNAs can Help in Bridging Psychiatry and Molecular Biology in the Context of Depression, Bipolar Disorder and Schizophrenia.[J]. Cellular and molecular neurobiology,2019,39(6):

47 Zuowei Wang,Chen Zhang,Jia Huang,et al. MiRNA-206 and BDNF genes interacted in bipolar I disorder[J]. Journal of Affective Disorders,2014,162: 
48 Howe James R,Li Emily S,Streeter Sarah E,et al. MiR-338-3p regulates neuronal maturation and suppresses glioblastoma proliferation.[J]. PloS one,2017,12(5):

49 Michael F. Egan,Masami Kojima,Joseph H. Callicott,et al. The BDNF val66met Polymorphism Affects Activity-Dependent Secretion of BDNF and Human Memory and Hippocampal Function[J]. Cell,2003,112(2):

50 Yukako Nakajo,Susumu Miyamoto,Yoshikazu Nakano,et al. Genetic increase in brain-derived neurotrophic factor levels enhances learning and memory[J]. Brain Research,2008,1241:

51 Moon Jangsup,Lee Soon-Tae,Kong II Gyu,et al. Early diagnosis of Alzheimer's disease from elevated olfactory mucosal miR-206 level.[J]. Scientific reports,2016,6:

52 Chih-Hua Chang,Elizabeth Joo Wen Kuek,Chun-Lin Su,et al. MicroRNA-206 Regulates Stress-Provoked Aggressive Behaviors in Post-weaning Social Isolation Mice[J]. Molecular Therapy - Nucleic Acids,2020,20:

53 Qian Qi,Zhang Jian,He Fang-Ping,et al. Down-regulated expression of microRNA-338-5p contributes to neuropathology in Alzheimer's disease.[J] .FASEB J, 2019, 33: 4404-4417.

54 Yuan Xiaojing,Wei Yujun,Ao Tianrang, et al. Effects of microRNA-338 Transfection into Sciatic Nerve on Rats with Experimental Autoimmune Neuritis.[J] .J Mol Neurosci, 2021, 71: 713-723.

55 Li Junhua,Li Danhua,Zhou Huatao,et al. MicroRNA-338-5p alleviates neuronal apoptosis via directly targeting BCL2L11 in APP/PS1 mice.[J] .Aging (Albany NY), 2020, 12: 20728-20742.

56 Liu Jia,Cao Linggai,Feng Yue, et al. MiR-338-3p inhibits TNF-a-induced lipogenesis in human sebocytes.[J]. Biotechnology letters,2017,39(9):

57 Nan Wang,Guang-Fen Zhang,Xiao-Yu Liu,et al. Downregulation of Neuregulin 1-ErbB4 Signaling in Parvalbumin Interneurons in the Rat Brain May Contribute to the Antidepressant Properties of Ketamine[J]. Journal of Molecular Neuroscience,2014,54(2):

58 Jinesh G Goodwin,Chunduru Srinivas,Kamat Ashish M. Smac mimetic enables the anticancer action of BCG-stimulated neutrophils through TNF-a but not through TRAIL and FasL.[J] .J Leukoc Biol, 2012, 92: 233-44.

59 Zhao Xueqiang,Rong Lijie,Zhao Xiaopu,et al. TNF signaling drives myeloid-derived suppressor cell accumulation.[J]. The Journal of clinical investigation,2012,122(11):

60 Chen Zhang,Li Kang,Haihui Zhu,et al. miRNA-338-3p/CAMK Ila signaling pathway prevents acetaminophen-induced acute liver inflammation in vivo[J]. Annals of Hepatology,2020: 
61 Sui Xiaofang, Yu Shuqian, Wang Fengling,et al. Effects of mir-338-3p overexpression on inflammatory signaling pathway [J]. Chinese journal of experimental diagnostics,2017,21(11):1991-1995.

62 Zhu X R,Wulf A,Schwarz M,et al. Characterization of human Kv4.2 mediating a rapidly-inactivating transient voltage-sensitive K+ current.[J]. Receptors \& channels,1999,6(5):

63 Dirk Isbrandt,Thorsten Leicher,Ralph Waldschütz,et al. Gene Structures and Expression Profiles of Three Human KCND (Kv4) Potassium Channels Mediating A-Type Currents I TO and I SA[J]. Genomics,2000,64(2):

64 Bähring R,Boland L M,Varghese A,et al. Kinetic analysis of open- and closed-state inactivation transitions in human Kv4.2 A-type potassium channels.[J] .J Physiol, 2001, 535: 65-81.

65 Patricia Mansfield,John N. Constantino,Dustin Baldridge. MYT1L : A systematic review of genetic variation encompassing schizophrenia and autism[J]. American Journal of Medical Genetics Part B: Neuropsychiatric Genetics,2020,183(4):

66 Barshir Ruth,Fishilevich Simon,Iny-Stein Tsippi,et al. GeneCaRNA: A Comprehensive Gene-centric Database of Human Non-coding RNAs in the GeneCards Suite[J]. Journal of Molecular Biology,2021,433(11):

67 Yanqing Shi,Qi Shao,Zhenghao Li,et al.Myt1L Promotes Differentiation of Oligodendrocyte Precursor Cells and is Necessary for Remyelination After Lysolecithin-Induced Demyelination[J].Neuroscience Bulletin,2018,34(02):247-260.

68 C Nagy,M Suderman,J Yang,et al. Astrocytic abnormalities and global DNA methylation patterns in depression and suicide[J]. Molecular Psychiatry,2015,20(3):

69 Xin Huang,Ling-ling Zhu,Tong Zhao,et al. CHL1 negatively regulates the proliferation and neuronal differentiation of neural progenitor cells through activation of the ERK1/2 MAPK pathway[J]. Molecular and Cellular Neuroscience,2011,46(1):

70 Yang C R,Ning L,Zhou F H,et al. Downregulation of Adhesion Molecule CHL1 in B Cells but Not T Cells of Patients with Major Depression and in the Brain of Mice with Chronic Stress.[J]. Neurotoxicity research,2020,38(4):

71 Katherine Najera,B. Matthew Fagan,Peter M. Thompson. SNAP-25 in Major Psychiatric Disorders: A Review[J]. Neuroscience,2019,420:

72 Alfredo Ramos-Miguel,Kristina Gicas,Jehan Alamri,et al. Beasley,Andrew J. Dwork,J. John Mann,Gorazd Rosoklija,Fang Cai,Weihong Song,Alasdair M. Barr,William G. Honer. Reduced SNAP25 Protein Fragmentation Contributes to SNARE Complex Dysregulation in Schizophrenia Postmortem Brain[J]. Neuroscience,2019,420: 
73 Sanja Selak,Ana V. Paternain,M. Isabel Aller,et al. A Role for SNAP25 in Internalization of Kainate Receptors and Synaptic Plasticity[J]. Neuron,2009,63(5):

74 Alba Cortés,Carla Muñoz-Antoli,J. Guillermo Estebanet al. Th2 and Th1 Responses: Clear and Hidden Sides of Immunity Against Intestinal Helminths[J]. Trends in Parasitology,2017,33(9):

75 Moaaz Mai,Youssry Sara,Elfatatry Amr,et al. Th17/Treg cells imbalance and their related cytokines (IL17, IL-10 and TGF- $\beta$ ) in children with autism spectrum disorder.[J] .J Neuroimmunol, 2019, 337: 577071.

76 Slyepchenko Anastasiya,Maes Michael,Köhler Cristiano A,et al. T helper 17 cells may drive neuroprogression in major depressive disorder: Proposal of an integrative model.[J]. Neurosci Biobehav Rev, 2016, 64: 83-100.

77 Fasching Patrizia,Stradner Martin,Graninger Winfried,et al. Therapeutic Potential of Targeting the Th17/Treg Axis in Autoimmune Disorders.[J] .Molecules, 2017, 22: undefined.

78 Beurel Eléonore,Harrington Laurie E,Jope Richard S,et al. Inflammatory T helper 17 cells promote depression-like behavior in mice.[J] .Biol Psychiatry, 2013, 73: 622-30.

79 Gopalsamy Rajiv Gandhi,Maria Terezinha Santos Leite Neta,Rajiv Gandhi Sathiyabama,et al. Flavonoids as Th1/Th2 cytokines immunomodulators: A systematic review of studies on animal models[J]. Phytomedicine,2018,44:

80 Markus Kleinewietfeld,David A. Hafler. The plasticity of human Treg and Th17 cells and its role in autoimmunity[J]. Seminars in Immunology,2013,25(4):

81 Schlaaff Konstantin,Dobrowolny Henrik,Frodl Thomas,et al. Increased densities of T and B lymphocytes indicate neuroinflammation in subgroups of schizophrenia and mood disorder patients[J]. Brain Behavior and Immunity,2020,88:

82 de la Peña Francisco Rafael,Cruz Fuentes Carlos,Palacios Lino,et al. Serum levels of chemokines in adolescents with major depression treated with fluoxetine.[J]. World journal of psychiatry,2020,10(8):

83 Pandey Ghanshyam N.,Rizavi Hooriyah S.,Bhaumik Runa,Zhang Hui. Chemokines gene expression in the prefrontal cortex of depressed suicide victims and normal control subjects[J]. Brain Behavior and Immunity,2021,94:

84 Ahmad SF, Ansari MA, Zoheir KM,et al. Regulation of TNF-a and NF-KB activation through the JAK/STAT signaling pathway downstream of histamine 4 receptor in a rat model of LPS-induced joint inflammation. Immunobiology. 2015 Jul;220(7):889-98.

85 Liu Yang,Zhang Jian,Zhou Yu-Hui,et al. Activation of the IL-6/JAK2/STAT3 pathway induces plasma cell mastitis in mice.[J] .Cytokine, 2018, 110: 150-158. 
86 Singh Ashok K,Bhadauria Archana S,Kumar Umesh,et al. Novel Indole-fused benzo-oxazepines (IFBOs) inhibit invasion of hepatocellular carcinoma by targeting IL-6 mediated JAK2/STAT3 oncogenic signals. [J] .Sci Rep, 2018, 8: 5932.

87 Zhang Hai-Yang,Jin Xun-Bo,Lue Tom F,et al. Three important components in the regeneration of the cavernous nerve: brain-derived neurotrophic factor, vascular endothelial growth factor and the JAK/STAT signaling pathway.[J] .Asian J Androl, 2011, 13: 231-5.

88 Ping Xin,Xiaoyun Xu,Chengjie Deng,et al. The role of JAK/STAT signaling pathway and its inhibitors in diseases[J]. International Immunopharmacology,2020,80:

89 Niu Liangliang,Fang Yuan,Yao Xiaoqian,et al. TNFa activates MAPK and Jak-Stat pathways to promote mouse Müller cell proliferation[J]. Experimental Eye Research,2020,202(prepublish):

90 Shirui Tan,Jian Xu,Aiyun Laiet,et al. Curculigoside exerts significant anti-arthritic effects in vivo andin vitro via regulation of the JAK/STAT/NF-KB signaling pathway[J]. Molecular Medicine Reports,2019,19(3):

91 Yeung Yiu To,Aziz Faisal,Guerrero-Castilla Angelica,et al. Signaling Pathways in Inflammation and Anti-inflammatory Therapies.[J]. Current pharmaceutical design,2018,24(14):

92 Hixson Kathryn M,Cogswell Meaghan,Brooks-Kayal Amy R,et al. Evidence for a non-canonical JAK/STAT signaling pathway in the synthesis of the brain's major ion channels and neurotransmitter receptors.[J]. BMC genomics,2019,20(1):

93 Ingrid V. Lund,Yinghui Hu,YogendraSinh H. Raol,et al. BDNF Selectively Regulates GABAA Receptor Transcription by Activation of the JAK/STAT Pathway[J]. Sci. Signal.,2008,1(41):

94 Robert Dantzer,Jason C. O'Connor,Gregory G. Freund,et al. From inflammation to sickness and depression: when the immune system subjugates the brain[J]. Nature Reviews Neuroscience,2008,9(Suppl 3):

95 Sian-Marie Lucas,Nancy J Rothwell,Rosemary M Gibson. The role of inflammation in CNS injury and disease[J]. British Journal of Pharmacology,2006,147(S1):

96 Li Qinzhi,Liu Yang,Wang Xiujuan,et al. Regulation of Th1/Th2 and Th17/Treg by pDC/mDC imbalance in primary immune thrombocytopenia[J]. Experimental Biology and Medicine,2021,246(15):

97 M L Molendijk,P Spinhoven,M Polak,et al. Serum BDNF concentrations as peripheral manifestations of depression: evidence from a systematic review and meta-analyses on 179 associations $(\mathrm{N}=9484)[\mathrm{J}]$. Molecular Psychiatry,2014,19(2):

98 Soo-Jeong Kim,Hyojung Lee,Gihyun Lee,et al. CD4+CD25+ regulatory T cell depletion modulates anxiety and depression-like behaviors in mice.[J]. PLoS ONE,2017,7(7): 
Figures

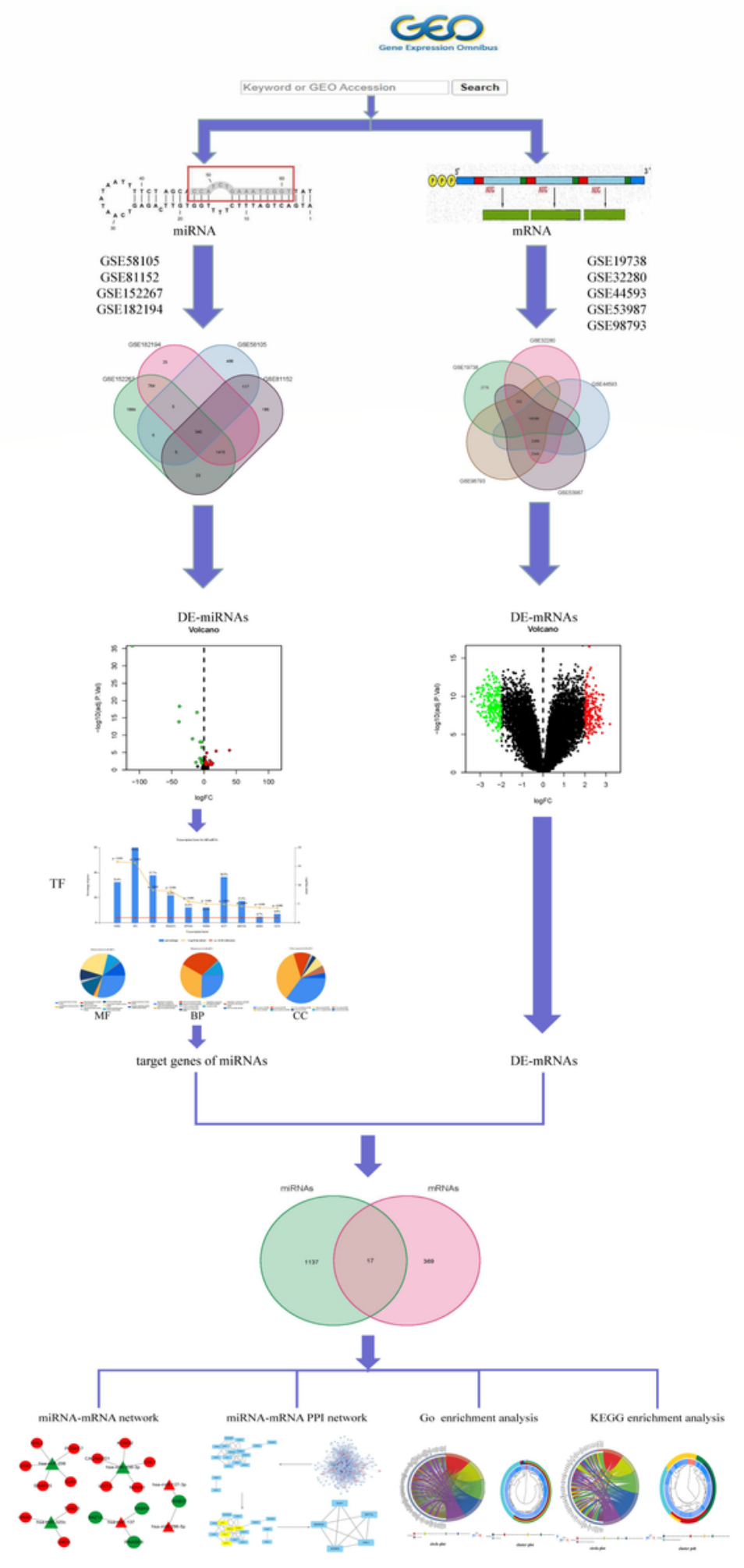

Figure 1

Framework based on an integration strategy of integrating analysis of miRNA-mRNA Regulatory Networks. 


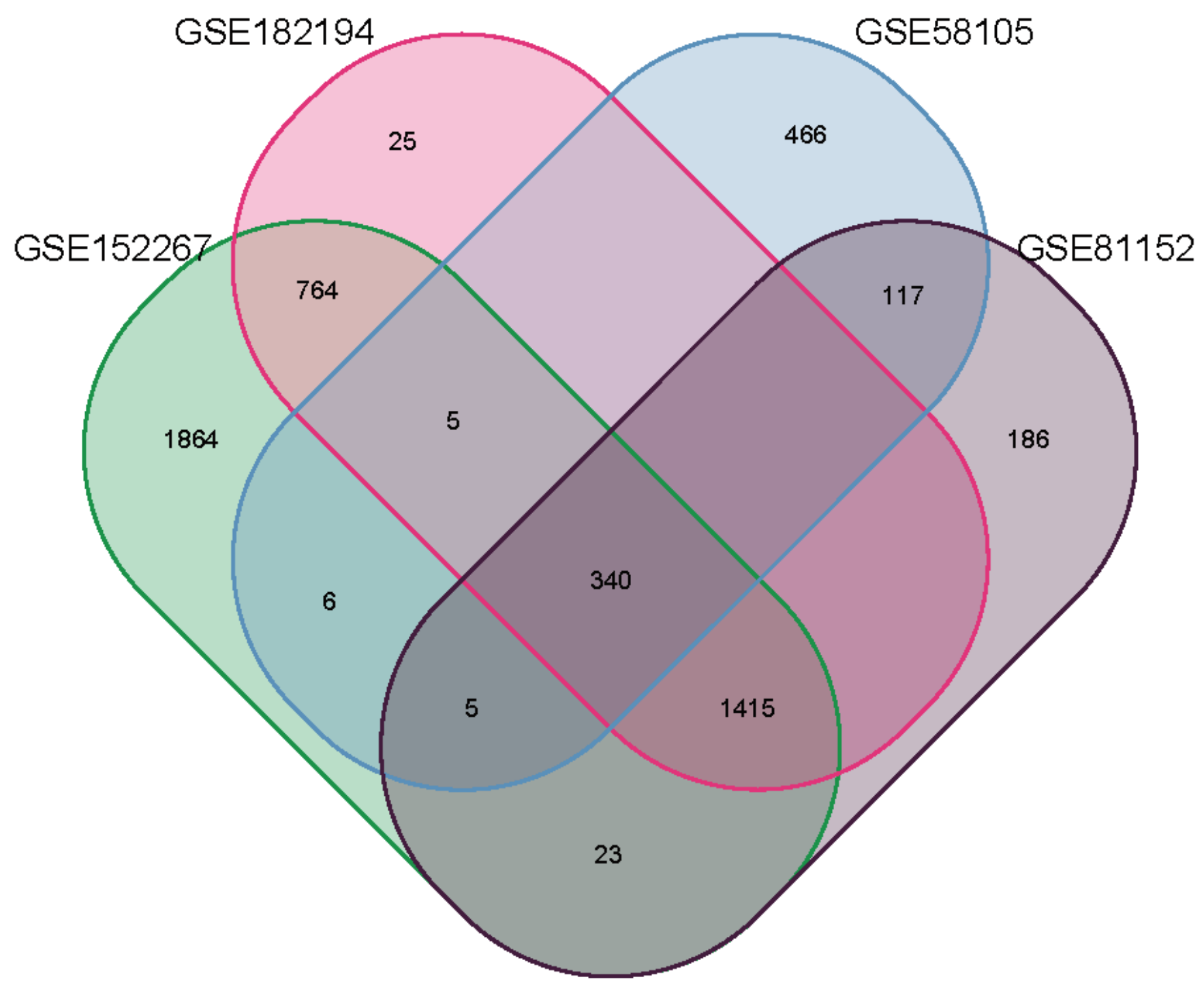

Figure 2

Venn diagram of miRNA of the merger GSE58105,GSE81152,GSE152267 and GSE182194. 


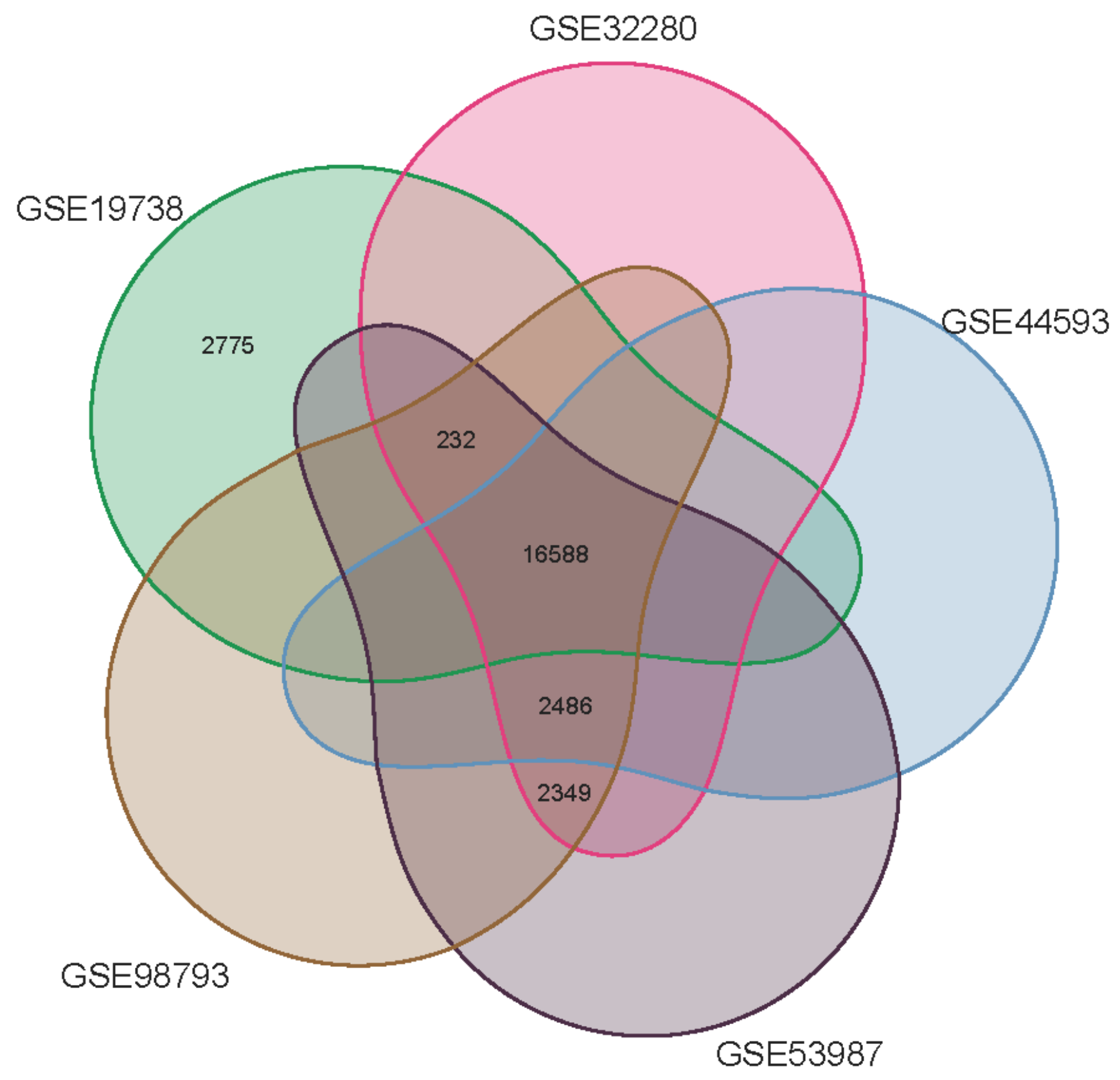

Figure 3

Venn diagram of mRNA of the merger GSE19738, GSE32280, GSE44593, GSE53987 and GSE98793. 

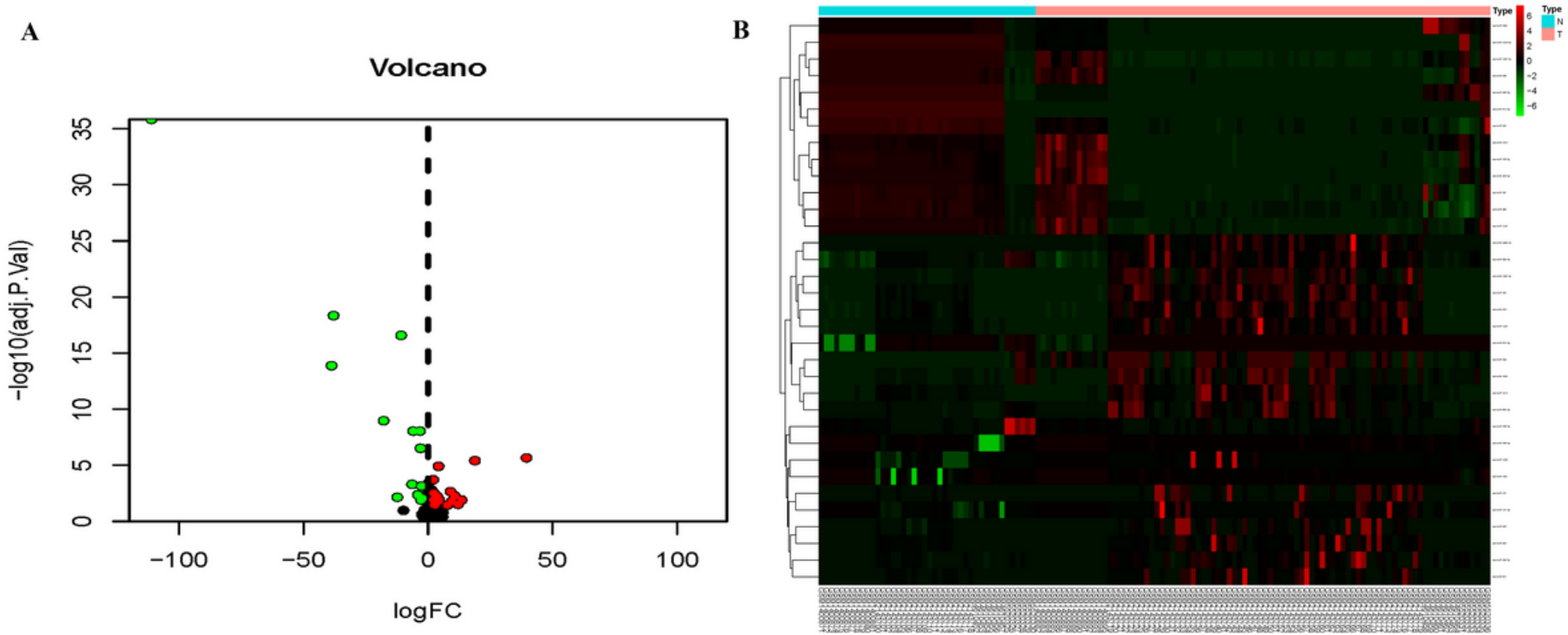

\section{Figure 4}

Differential genes volcano map and heat map are jointly analyzed by 4 miRNA chips. miRNA from normal group and MMD group.

A

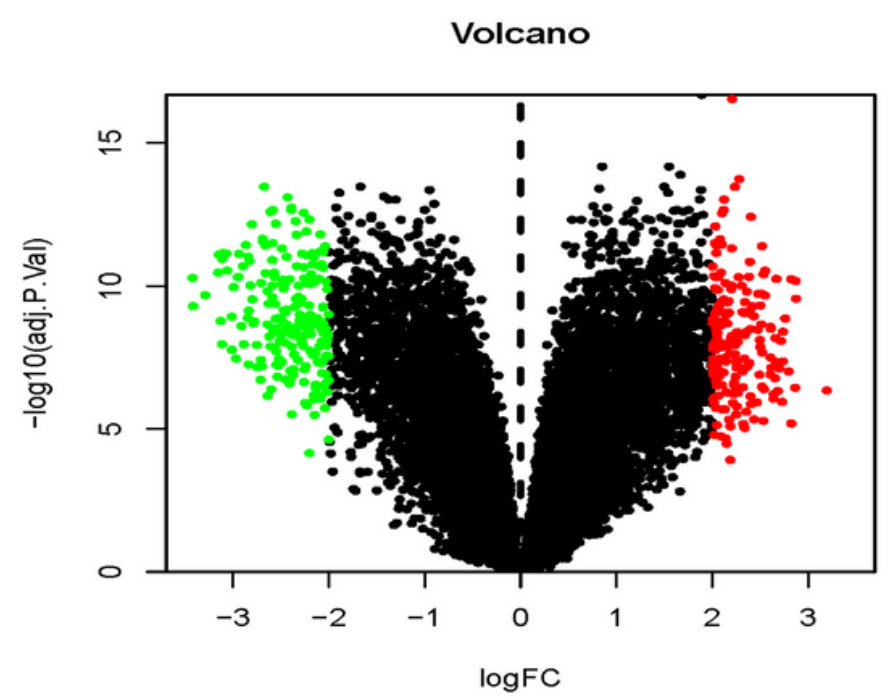

B

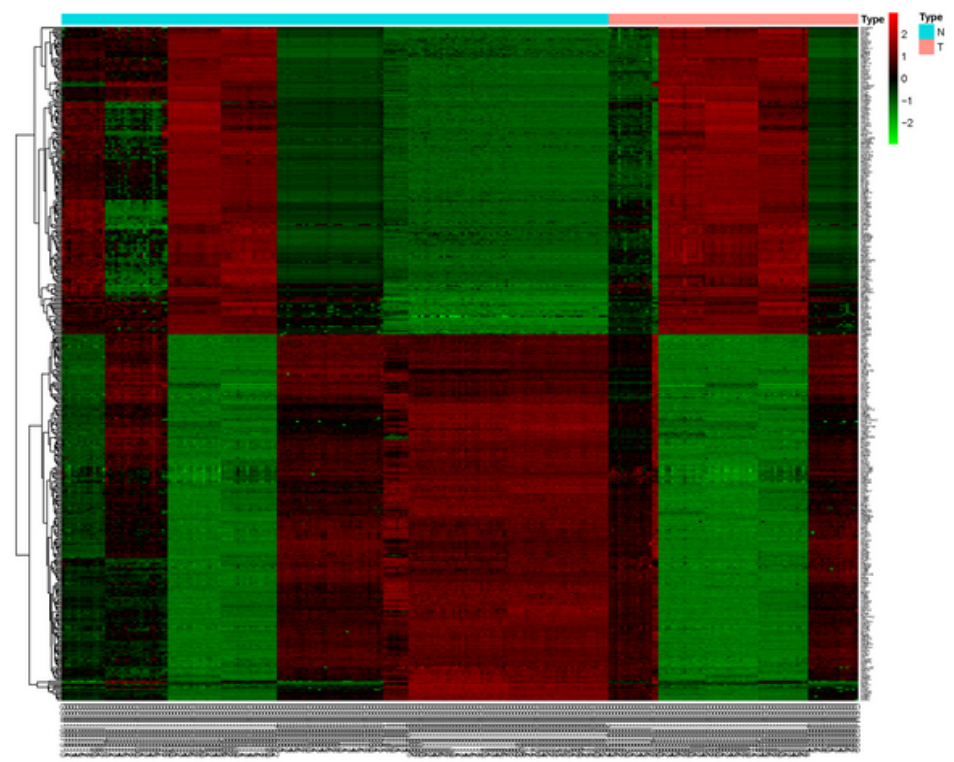

Figure 5

Differential genes volcano map and heat map are jointly analyzed by 5 mRNA chips. mRNA from normal group and MMD group. 

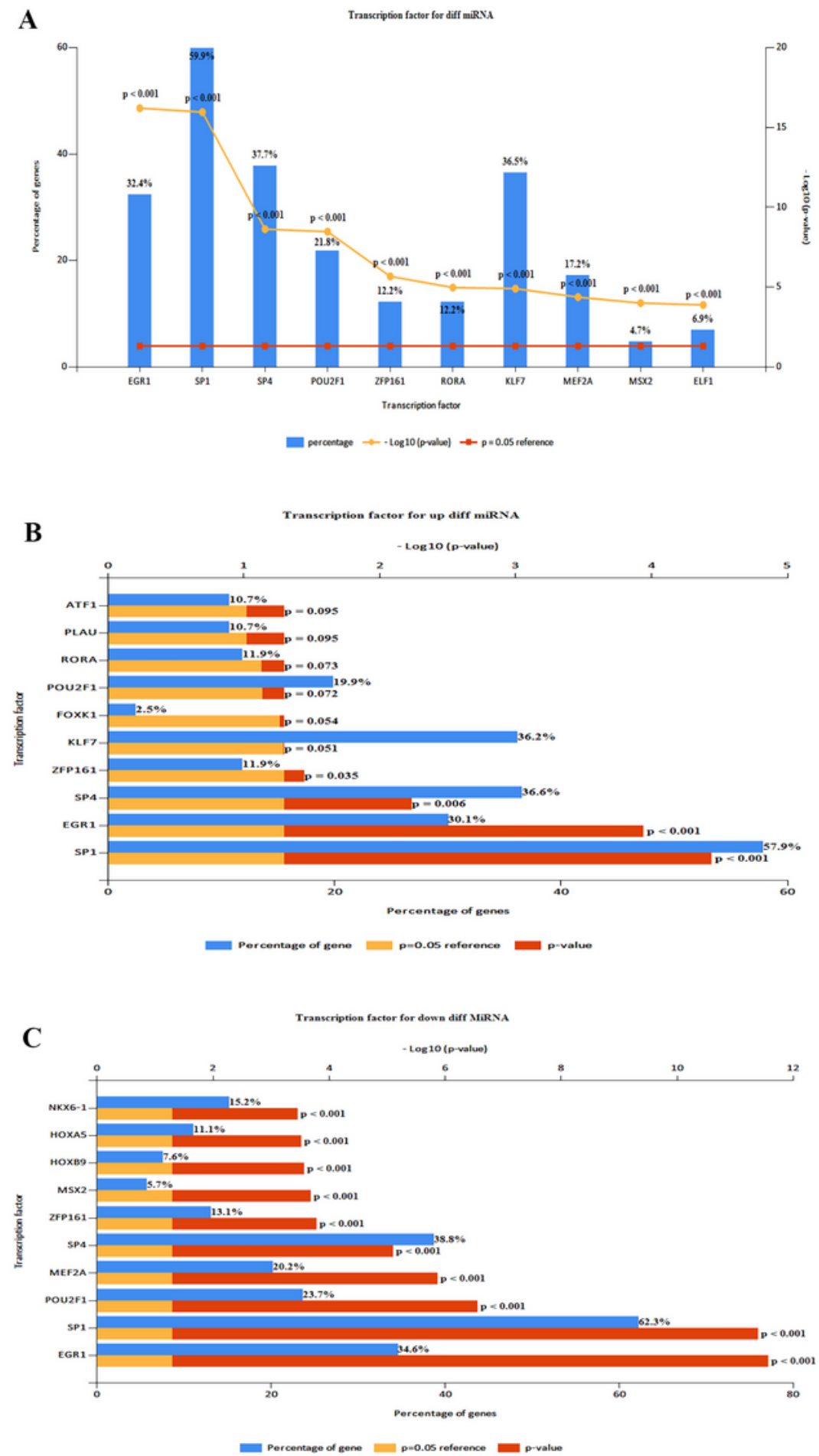

\section{Figure 6}

(A)Top 10 TF of DE-miRNAs. (B) Top 10 TF of up-regulated DE-miRNAs. (C) Top 10 TF of down-regulated DE-miRNAs.. 
Molecular function for diff miRNA
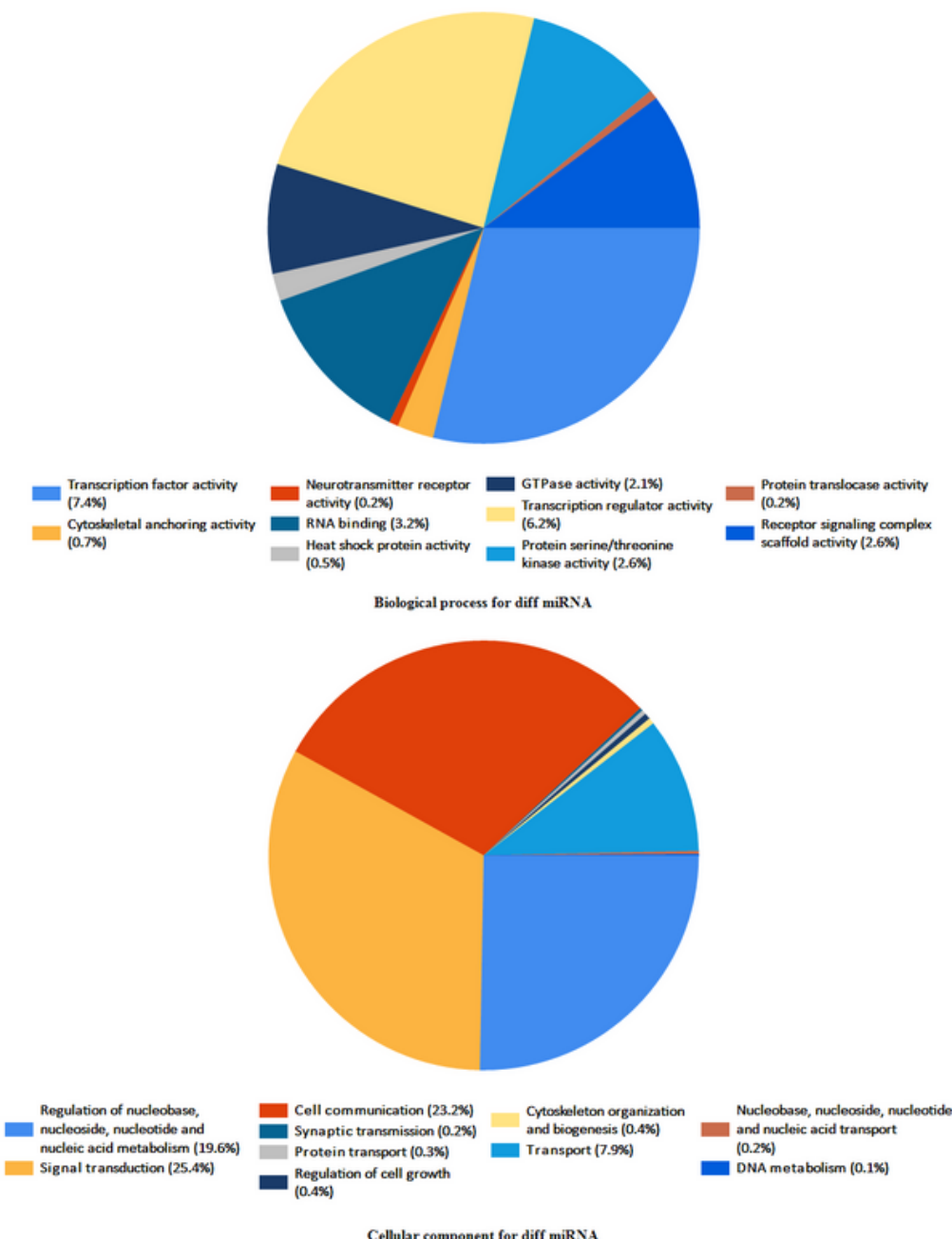

Cellular component for diff miRNA

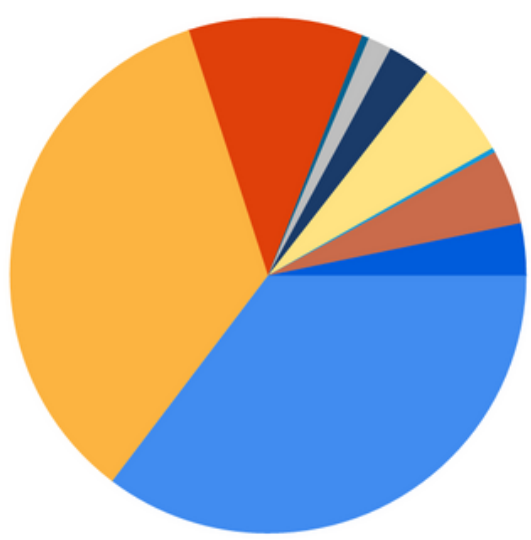

Cytoplasm (49.3\%) Dysosome (15.2\%) Actin eytoskeleton (2.1\%) Golgi aparatus (8.7\%) Centrosone (6.5\%) Nucleus (48.4\%) $\begin{aligned} & \text { Apical membrane (0.6\%) } \\ & \text { Endosome (3.7\%) }\end{aligned}$

\section{Figure 7}

GO enrichment analysis of DE-miRNAs. 


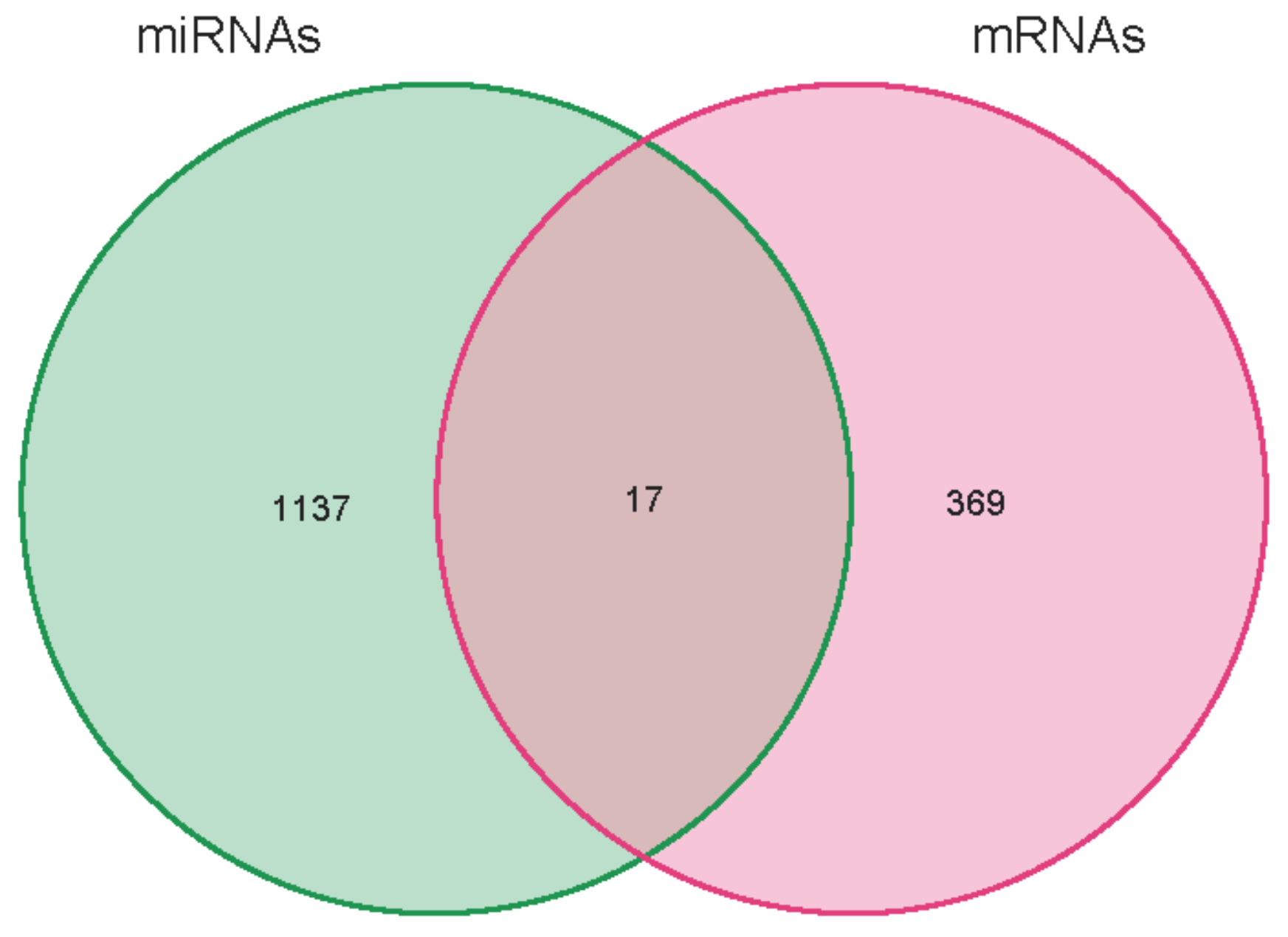

Figure 8

Venn diagram of overlapping DE-mRNAs. 


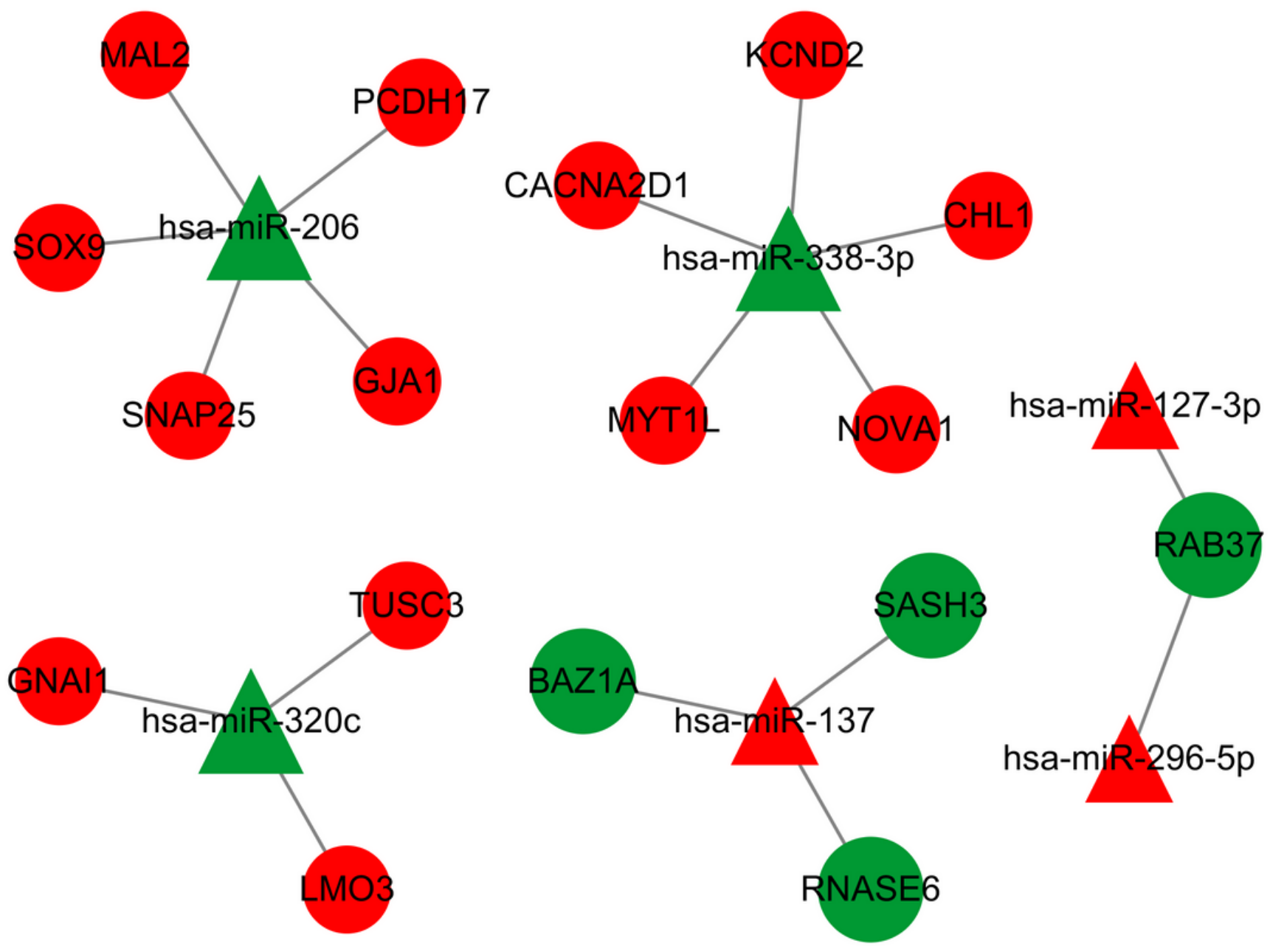

Figure 9

The miRNA-mRNA networks of all dysregulated mRNAs. Red stands for up-regulation, green for downregulation, triangle for miRNA, and prototype for mRNA. 

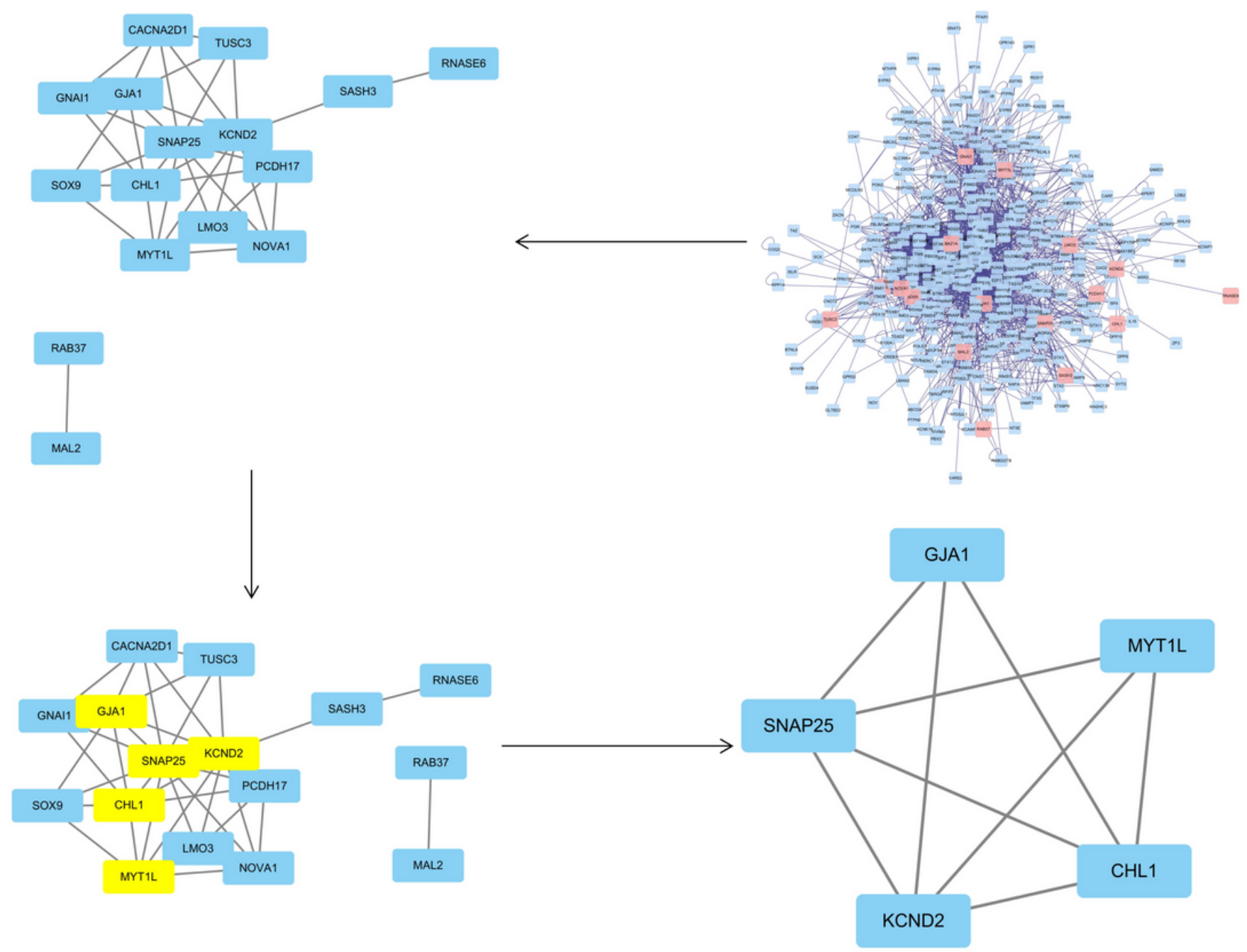

Figure 10

The process of topological screening for the PPI network. 


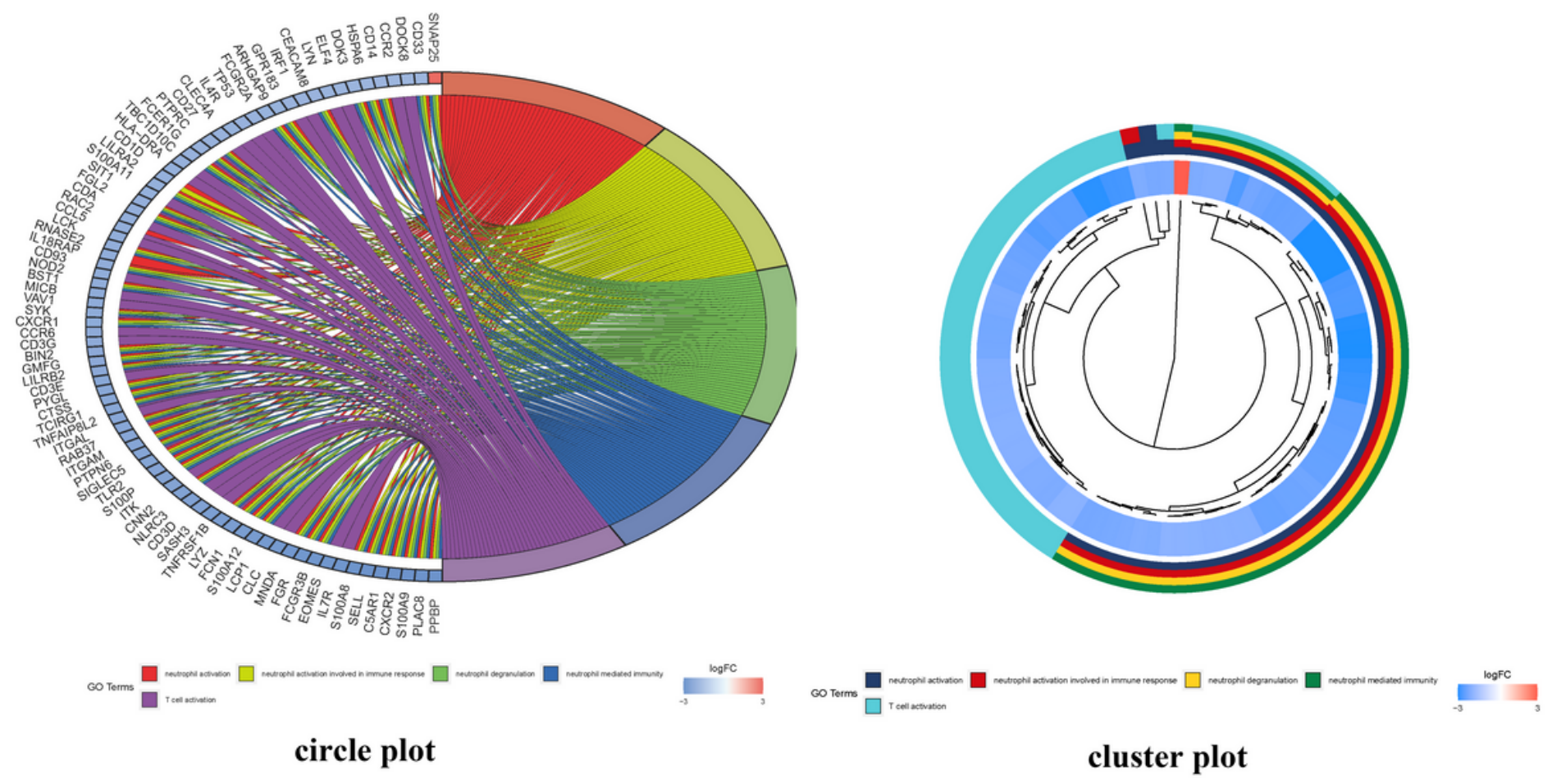

\section{Figure 11}

The GO enrichment analysis of core nodes. Including cellular components, molecular functions, biological processes
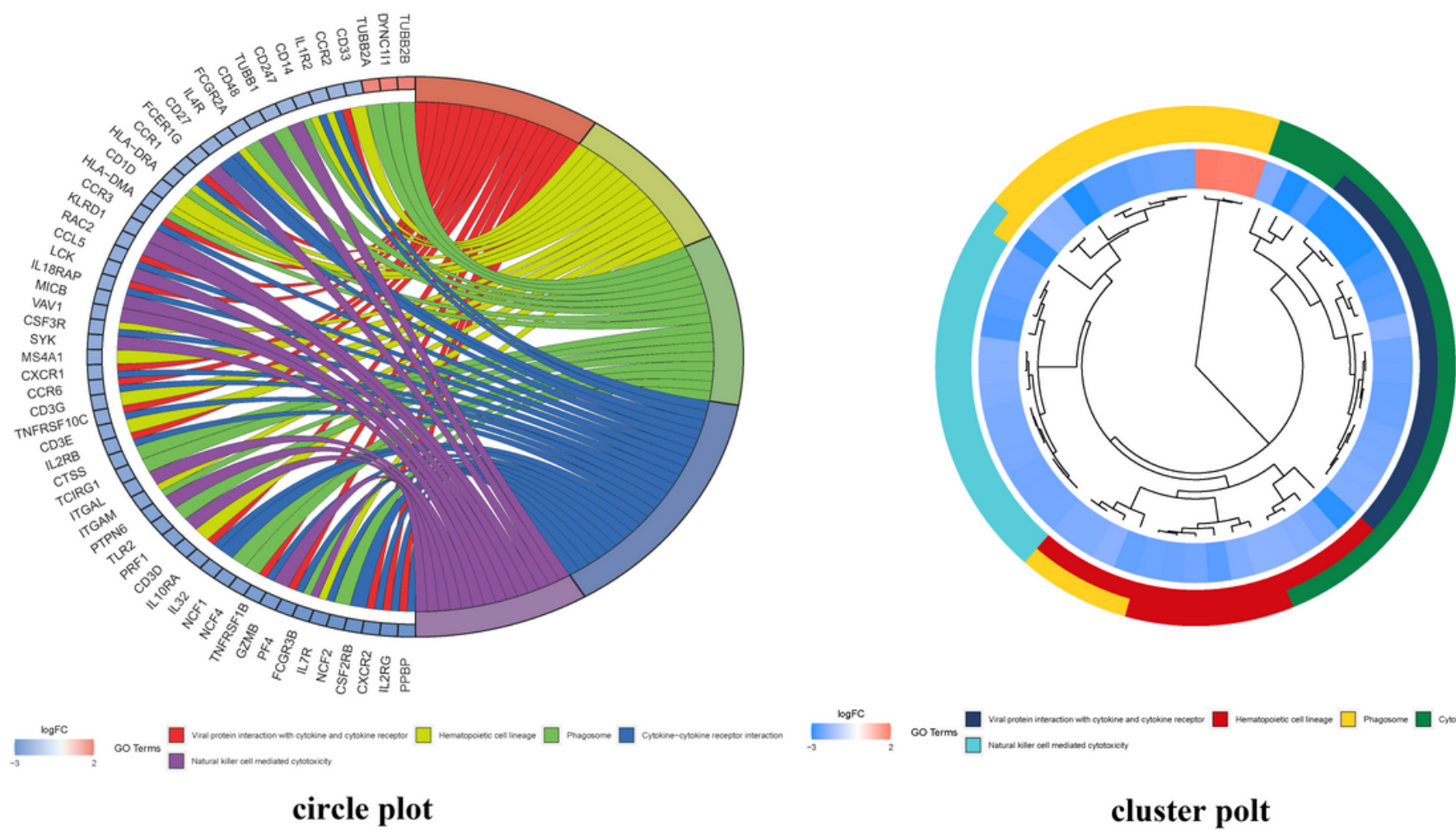

\section{cluster polt}

Figure 12 
The KEGG enrichment analysis of core nodes.

\section{Supplementary Files}

This is a list of supplementary files associated with this preprint. Click to download.

- appendix.doc 